Title: Controlling of compliant grinding for low-rigidity components

Authors:

Yue Yang ${ }^{a}$, Haonan Li ${ }^{a}$, Zhirong Liao ${ }^{b}$, Dragos Axinte ${ }^{b, a,}{ }^{*}$, Wule Zhu ${ }^{c}$, Anthony Beaucamp ${ }^{c}$

Affiliation:

a Faculty of Science and Engineering, University of Nottingham Ningbo China, Ningbo 315100, China

b Faculty of Engineering, University of Nottingham, Nottingham NG7 2RD, UK

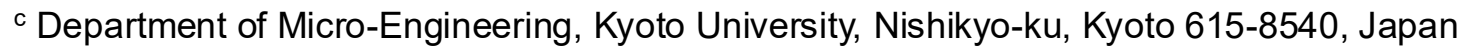

${ }^{*}$ Corresponding author: dragos.axinte@nottingham.ac.uk 
Highlights:

- A theoretical contact model for the first time was proposed for grinding involving two compliant bodies.

- The model reveals the position-variant characteristics of the relation between nominal and actual tool offsets.

- This paper proposed a new control strategy by time-variant tool offset design to obtain a constant material removal rate.

- The ground surfaces obtained from the proposed strategy reach the desired removal depth with an average error of $\leqslant 10 \%$. 


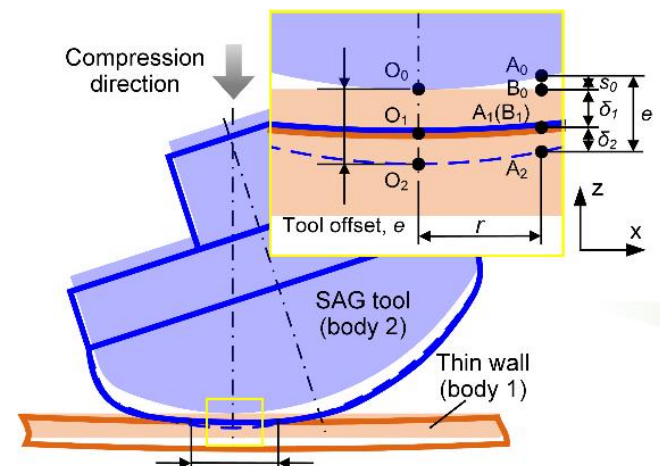

$\overrightarrow{\text { Contact area, } 2 a}$

Contact problem between two

compliances

Experimental setup

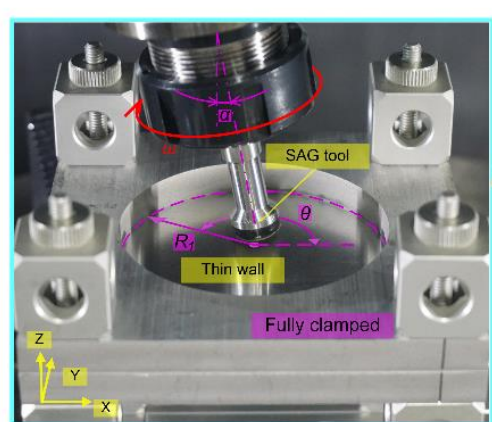

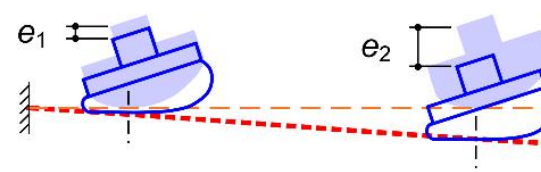

Theoretical model

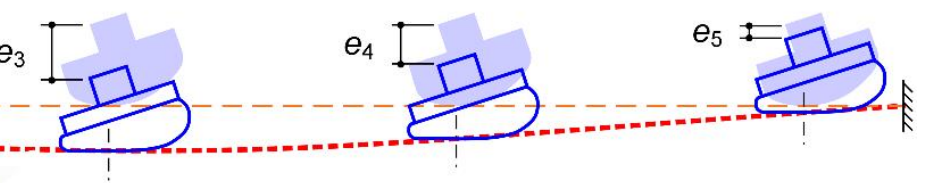

Time-variant tool offset $\left(e_{1} \neq e_{2} \neq e_{3} \neq e_{4} \neq e_{5}\right)$
Control strategy for constant material removal rate

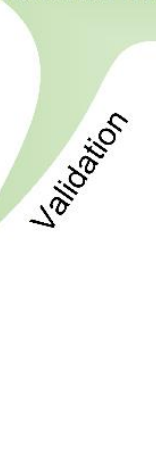

${ }^{0 / 3 / 3 / 8}$

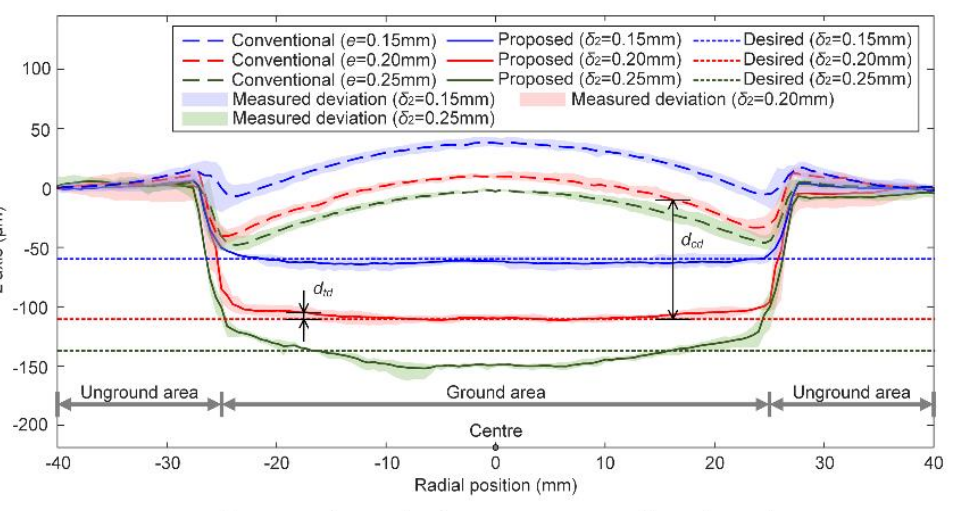

Comparison between conventional and proposed strategies 


\title{
Controlling of compliant grinding for low-rigidity components
}

\author{
Yue Yanga, Hao Nan Lia, Zhirong Liaob, Dragos Axinte ${ }^{b, a,{ }^{*}}$, Wule Zhu'c, Anthony Beaucamp ${ }^{c}$ \\ a Faculty of Science and Engineering, University of Nottingham Ningbo China, Ningbo, China \\ ${ }^{b}$ Faculty of Engineering, University of Nottingham, Nottingham, UK \\ ${ }^{c}$ Department of Micro-Engineering, Kyoto University, Kyoto, Japan
}

\begin{abstract}
:
The machining of low-rigidity components (e.g. thin-walled) with compliant tools presents accuracy challenges as both sides in contact are being deformed. The controlling method presented in this paper enables, for the first time, to obtain the desired and uniform material removal rate by controlling the nominal tool offset when two bodies (workpiece and tool) are compliant in grinding. A contact deformation model is proposed to predict the relation between the nominal and actual tool offsets. The function of nominal tool offsets and material removal rates is obtained based on the calibration tests. Spot grinding tests have been performed for the validation of the calculated material removal rates, normal grinding forces and spot sizes, presenting position-dependent characteristics. The controlling method has been tested for the case of continuous grinding the whole area of a circular aluminium thin wall. The surfaces ground under the time-variant tool offsets (proposed approach) reach the desired removal depth with an average error of $\leq 10 \%$ and achieve $11.2 \mu \mathrm{m} \sim 24.2 \mu \mathrm{m}(\mathrm{P}-\mathrm{V})$ accuracy in the elastic domain, compared with the error of $76.8 \% \sim 113.7 \%$ and accuracy of $42.6 \mu \mathrm{m} \sim 50.1 \mu \mathrm{m}$ $(\mathrm{P}-\mathrm{V})$ in the circumstance of constant tool offsets (conventional approach).
\end{abstract}

Keywords: compliant grinding, material removal rate, tool offset, thin wall machining

\section{Nomenclature}

$\begin{array}{ll}\text { A } & \text { coefficient matrix }\left(\mathrm{mm}^{3} / \mathrm{N}\right) \\ a, a_{c} & \text { radii of the contact region and candidate one }(\mathrm{mm}) \\ B & \text { compliant motion matrix }(\mathrm{mm}) \\ b & \begin{array}{l}\text { distance between the grinding position and thin wall centre }(\mathrm{mm}) \\ \text { constants of integration }\end{array} \\ C_{1,2,3} & \begin{array}{l}\text { bending stiffness of the thin wall }\left(\mathrm{N}^{*} \mathrm{~mm}\right) \\ D_{1}\end{array} \\ d_{c d}, d_{d}, d_{t d} & \begin{array}{l}\text { distance between the case of constant tool offset and the desired depth, } \\ \text { desired depth, distance between the case of time-variant tool offset and the } \\ \text { desired depth }(\mu \mathrm{m})\end{array}\end{array}$




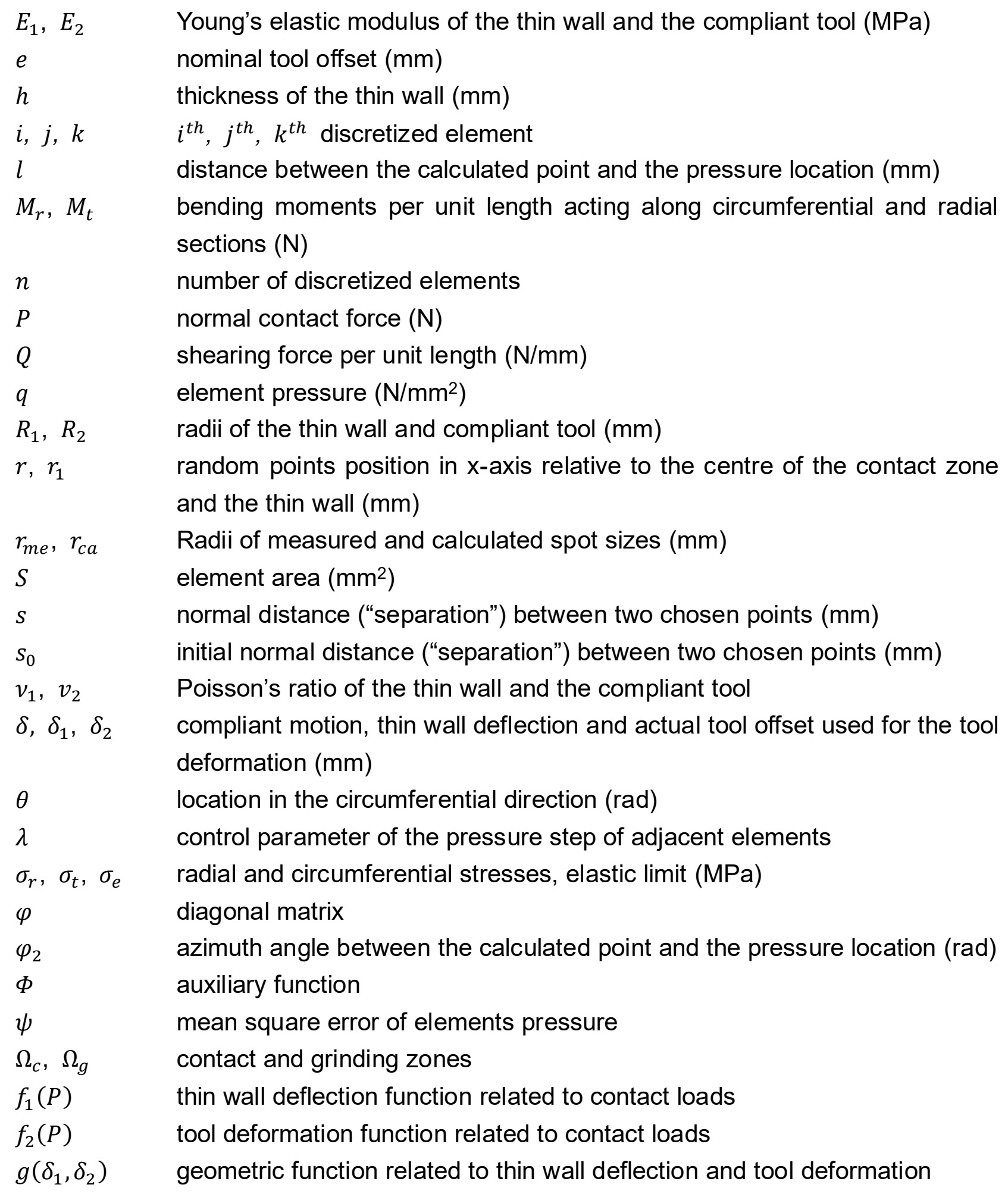

\section{Introduction}

With the increased demand for smooth surfaces on thin-walled components with high accuracy in aerospace, biomedical, electronics and automotive engineering, the development of the grinding techniques merits special attention. Conventional mechanical 
grinding tools, are mostly considered as rigid composite bodies [1] , such as grinding wheels, composed of bond agents (vitrified/resin/metallic) and abrasive grains $\left(\mathrm{SiC} / \mathrm{Al}_{2} \mathrm{O}_{3} /\right.$ diamond/CBN). Most ground workpiece also shows the infinite stiffness with no bending/distortion/ displacement occurring in the interaction with the tool [2,3]. Therefore, the material removal, surface profile and surface integrity are analyzed on the basis of the grinding processes with two rigid bodies (workpiece and tool) [4,5].

Although most machining operations are dealing with two rigid bodies (workpiece and tool), in thin-walled part machining, the tool is considered rigid while the workpiece is considered deformable due to the high ratios of the in-plane dimension to the thickness (more than ten [6]) which leads to low stiffness. Hence the amount of material removal is not constant because of the compliance of the workpiece. Generally, achieving precision machining of thin-walled parts is contingent on error compensation $[7,8]$. In such cases, the thin wall deflections from predictions considering mechanical/thermal loads $[9,10]$ or real-time online measurement $[8,11,12]$, are mostly used as position-dependent feedback to reselect and design processing parameters (e.g. smart tool paths [13-15] or constant contact forces [16]). However, these approaches solved the case in which only the workpiece is compliant.

There is another case where the tool is compliant, and the part is rigid, namely compliant grinding. More complexities than other grinding approaches are that the cutting mechanism of this process has characteristics of time dependence and compliance. The material removal in compliant grinding is measured by influence functions, which is another area of complexities because they depend on pressure distributions and relative velocities between the tool and workpiece as well as interfacial conditions based on Preston's Law [17]. Shape adaptive grinding (SAG) [18-20] and bonnet polishing tools [21], as two types of compliant grinding tools, show high shape adaptability in grinding sculptured freeform surfaces thanks to the tools' elastic substrate (rubber/air inflated). Mainly the compliant tools have been used for grinding rigid bodies (metallic/ceramic workpiece without macro deformations in contact with tools) [22-24] based on elastic contact forces predictions [25,26] and the modifications of the interfacial friction coefficient $[27,28]$. However, with this process being so versatile, grinding low-rigidity components with such tools should be studied, such as thin walls which are widely used in the engineering profession for aerospace and other scenarios where weight and cost are main considerations.

To the authors' best knowledge, there are very few attempts about two compliant elements being in contact, i.e. both the tool and workpiece, in relation to a mechanical material removal process. Similar studies (e.g. belt grinding of aero-engine blades) are normally based on 
scanning systems, calibration strategies and force sensors [29,30]. This paper takes advantages and interests on the complexity of SAG tools to grind the thin wall surfaces where both are compliant.

\subsection{Problem identification}

This paper reports on an attempt to deterministically obtain a desired constant material removal rate by the control of tool offsets in grinding processes where two soft bodies are involved, i.e. low-rigidity workpiece and shape adaptive grinding tool. In compliant grinding, the interaction area between the two bodies is dependent on the geometrical tool offset. The tool offset is given as the rigid motion distance along the normal direction of ground surfaces, starting from the initial contact point as the tool is compressed into the workpiece.

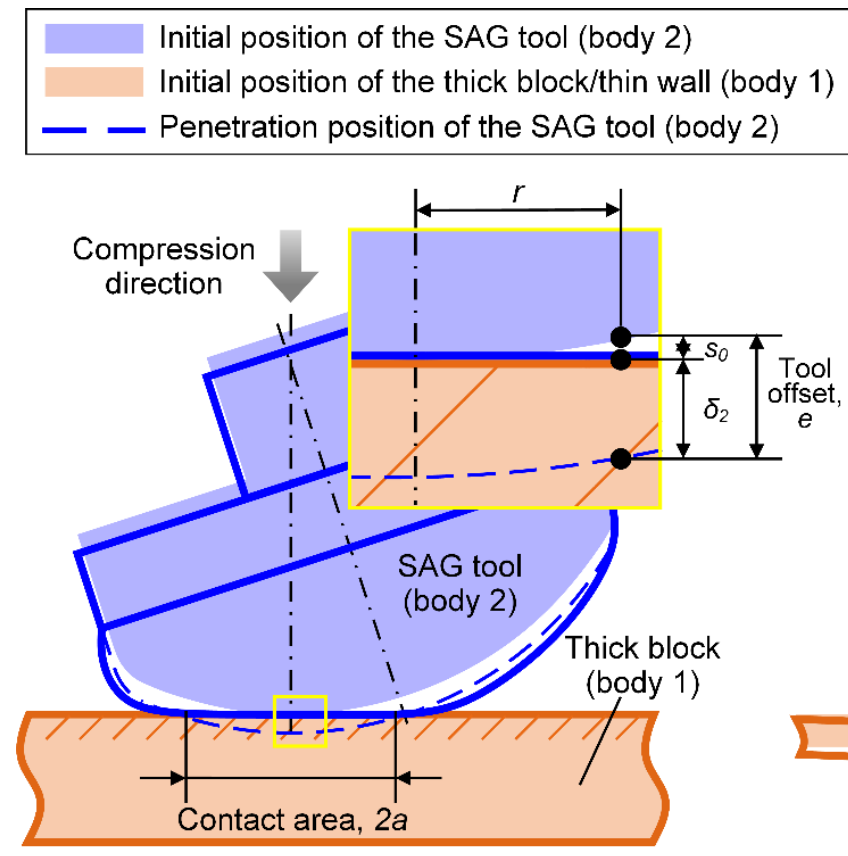

(a)
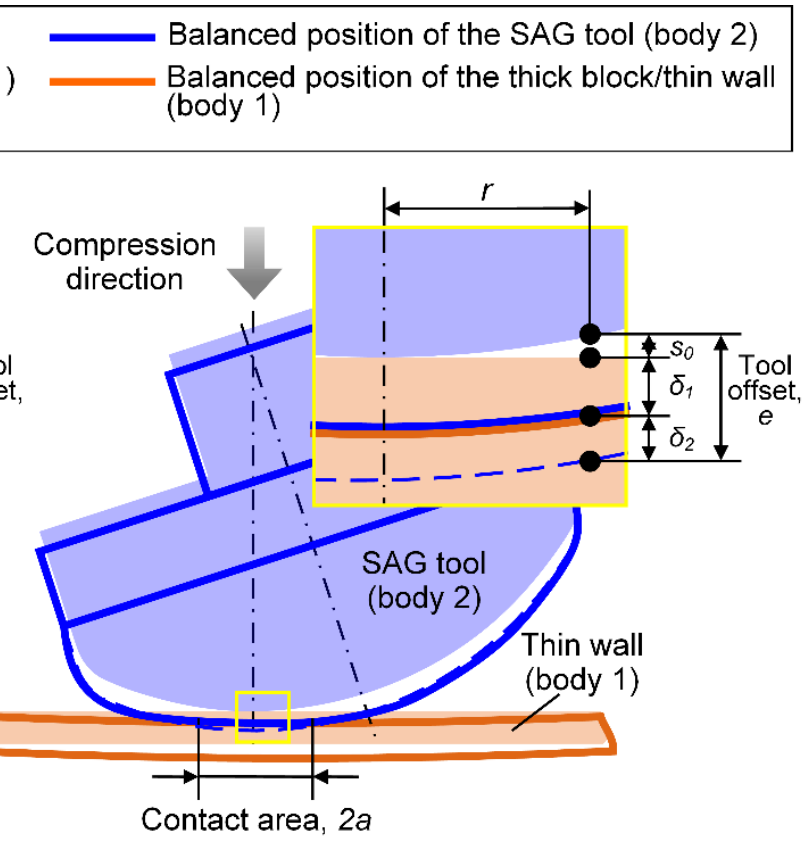

(b)

Fig. 1. Schematics of compliant grinding on thick blocks (a) and thin walls (b) enphasising key differences between phenomena occurring at the tool-workpiece contact zone.

The nominal tool offset is the key control parameter to obtain a constant material removal. It is used for the tool deformation in grinding rigid components, e.g. thick blocks (see in Fig. 1a). However, the nominal tool offset introduces a challenge in compliant grinding of thin walls because two soft bodies are both deformed under the given pressure until a new equilibrium state is reached, yielding a smaller tool deformation than in the case of grinding thick/rigid blocks. Specifically, the actual tool offset ( $\delta_{2}$ in Fig. 1b) used for deforming the tool is smaller than the nominal tool offset $(e$ in Fig. $1 \mathrm{~b})$ due to the thin wall deflections ( $\delta_{1}$ in Fig. 1b). Since 
the nominal tool offset is the only controllable factor for adjusting and compensating the thin wall deflections, the control strategies of tool offsets are the focus of this study based on the analysis of the SAG tool deformation and the thin wall deflection.

\section{Controlling process model}

This section develops the theoretical model for controlling the tool offset with the aim of achieving a constant material removal rate. The design of the tool offset is based on i) the relationship between the nominal and actual tool offsets (see section 2.1); ii) the control of tool offsets in shape adaptive grinding thin walls (see section 2.2); iii) the relationship between the actual tool offset and the material removal rate (see section 3.2).

\subsection{Contact deformation model}

For the aim of finding out the contact deformations of two elastic solids in the normal direction (the relationship between the given and the actual tool offsets), the problem can be depicted as the normal frictionless contact between an elastic sphere and a thin wall although there is relative motion at the interface between them due to the spindle rotation and feed movement. This is because, in the context of grinding, the geometry and kinematics of the process affect the shear in an abrasive contact, while the normal contact loads are not influenced by the process kinematics and shear forces [31]. The normal contact deformations are mainly dependent on the normal contact stresses.

To simplify this problem, the following assumptions are made:

- The contact pressure is assumed axi-symmetrically distributed within a circular region as the elastic substrate shape of the compliant tool is a partial sphere.

- The middle plane of the thin wall in the direction of thickness remains neutral during bending. Points initially perpendicular to the middle plane remain perpendicular to the deformed middle surface (Kirchhoff hypothesis).

- The thin wall is isotropic and deformed without the consideration of thermal effects due to slow cutting speeds and extremely low material removal rates.

- The thickness of thin walls remains unchanged as the compliant grinding is a fine finishing process with a very small grinding allowance.

The discrepancy between programmed and actual tool offset stems from different properties of the tool and workpiece in elastic deformation, i.e. stiffness of two elastic bodies. The elasticity of the SAG tool (body 2) is from the nature of its material (rubber substrate), while the flexibility of the thin wall (body 1 ) is dependent on its dimensions (i.e. ratio between length and thickness) and mechanical properties (i.e. Young's modulus and Poisson ratio). When a 
tool offset is given, it introduces the contact loads between two bodies. However, with the same pressure, two bodies react differently leading to unequal deformations. That means this normal frictionless contact problem is formulated to obtain the solution of the normal contact loads between two bodies under a nominal tool offset. It is considered that the normal frictionless contact is not affected by the surface roughness of the contact interfaces. The evolution equation of nominal tool offset $(e)$ can be expressed as

$$
e=g\left(\delta_{1}, \delta_{2}\right)=g\left(f_{1}(P), f_{2}(P)\right)
$$

where: $\delta_{1}$ - thin wall deflection; $\delta_{2}$ - actual tool offset used for tool deformations in the compressed direction; $P$ - contact load between two elastic bodies; $g\left(\delta_{1}, \delta_{2}\right)$ - geometric function related to thin wall deflection and actual tool offset; $f_{1}(P)$ - thin wall deflection function related to contact loads; $f_{2}(P)$ - actual tool offset function related to contact loads.

\subsubsection{The geometric relationship between nominal tool offset and two bodies deformation}

The geometric equation is depicted by the function $g\left(\delta_{1}, \delta_{2}\right)$ in Eq. (1). For two arbitrary points located on the two solids with the same position in the x-axis (see in Fig. 1b), the "separation" $(s)$ between two points after compression includes: i) initial distance $\left(s_{0}\right)$ where two bodies just contact without pressure, ii) rigid motion $(e)$, and iii) compliant motion ( $\delta_{1}$ and $\left.\delta_{2}\right)$.With regards to those that are within the contact region $\left(\Omega_{c}\right)$, the "separation" $(s)$ is zero. $g\left(\delta_{1}, \delta_{2}\right)$ can be described by

$$
\begin{gathered}
e=g\left(\delta_{1}, \delta_{2}\right)=s_{0}+\delta_{1}+\delta_{2} \\
s_{0}=R_{2}-\sqrt{R_{2}^{2}-r^{2}}, 0 \leq r \leq a
\end{gathered}
$$

where: $r$ - position of chosen two points projected to the $\mathrm{x}$-axis relative to the centre point $\mathrm{O}$; $R_{2}$ - radius of the SAG tool (body 2); $a$ - radius of the contact region.

The compliant motion $\left(\delta=\delta_{1}+\delta_{2}\right)$, i.e. the sum of thin wall deflection and the actual tool offset used for the tool deformation, varies with distances to the contact centre ( $r$ in Fig. 1b) in the contact zone, although the rigid motion is the same for each point on the surfaces. This is because the initial distances $\left(s_{0}\right)$ between the tool and workpiece are different. Meanwhile, the distributions/allocations of tool and workpiece deformations are different concerning different positions as mentioned above. It means that the solution of Eq. (1) should satisfy the equilibrium with respect to random positions in the contact zone. The discretization can approximate the equilibrium in the continuum by a finite number of parameters. After the contact zone was discretized, the equilibrium was established concerning centroids and nodes of elements that satisfy Eq. (1). In this way, the normal interaction forces between the 
two bodies are denoted by changing pressure distribution along the radial direction but the same within each discretized annular element.

$$
P=\sum_{i=1}^{n} P_{i}=\sum_{i=1}^{n} q_{i} S_{i}
$$

where: $P$ - normal contact force; $n$ - number of the discretized elements; $P_{i}$ - resultant contact force (pressure) in $i^{\text {th }}$ element; $q_{i}$ and $S_{i}$ - pressure and area of $i^{\text {th }}$ element; Details about discretization are shown in Appendix $A$.

\subsubsection{The function linking thin wall deflection and pressure}

For a thin wall (with a high ratio of length to thickness), the contact region is much smaller than in-plane dimensions of the thin wall, and therefore, to simplify this problem, we assumed the contact load is a concentrated force $(P)$ for the thin wall. The equation of $\delta_{1}=f_{1}(P)$ is calculated by considering two cases: $\mathrm{i}$ ) symmetric loading when the concentrated force $(P)$ is exerted at the centre of the thin wall (see in Fig. 2a), and ii) asymmetric loading when the concentrated force $(P)$ is exerted at eccentric positions relative to the thin wall centre (see in Fig. 2b); in both conditions, the geometry and boundary conditions (i.e. circular shape with fully clamped edges) are axisymmetric.

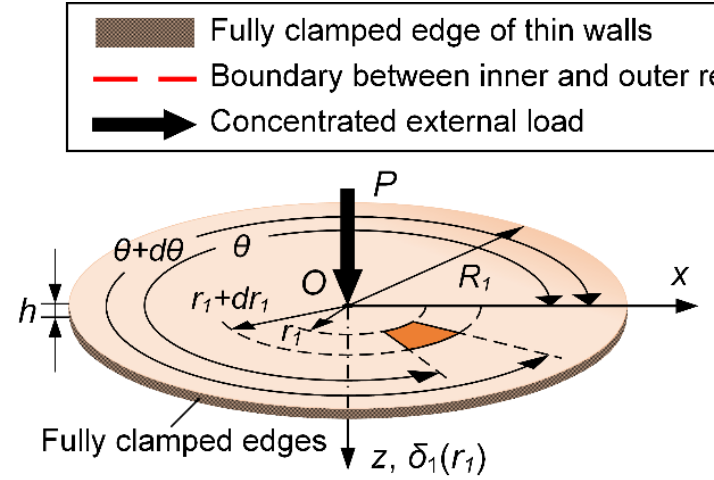

(a)

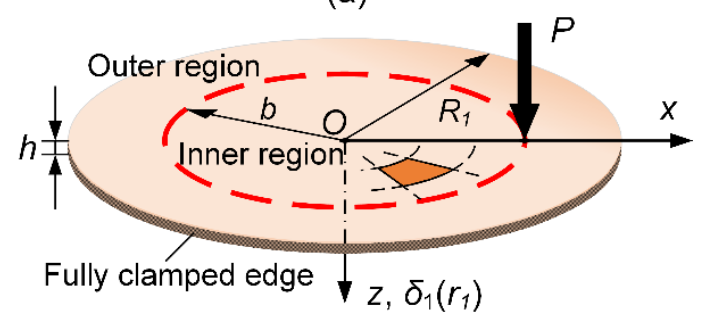

(b)

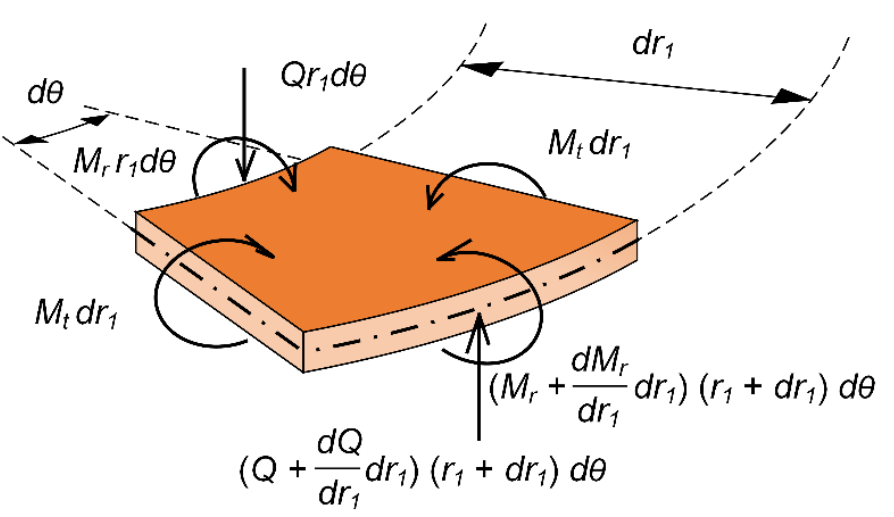

(c)

Fig. 2. Force analysis of the discretized element (c) when a concentrated load is symmetrically (a) and asymmetrically (b) exerted on the clamped circular thin wall. [6]

(i) Symmetric loading. When the grinding position is at the centre of the circular thin wall (see in Fig. 2a), bending moment equilibrium of an element (see in Fig. 2c) in the 
circumferential direction is [32]

$$
\left[M_{r}+\left(d M_{r} / d r_{1}\right) d r_{1}\right]\left(r_{1}+d r_{1}\right) d \theta-M_{r} r_{1} d \theta-M_{t} d r_{1} d \theta+Q r_{1} d \theta d r_{1}=0
$$

Simplifying, so it can be

$$
M_{r}+\left(d M_{r} / d r_{1}\right) r_{1}-M_{t}+Q r_{1}=0
$$

where: $M_{r}$ and $M_{t}$ - bending moments per unit length acting along circumferential and radial sections; $Q$ - the shearing force per unit length; $r_{1}$ and $\theta$ - the location in radial and circumferential directions.

$$
\begin{gathered}
M_{r}=-D_{1}\left[d^{2} \delta_{1} / d r_{1}^{2}+\left(v_{1} / r_{1}\right)\left(d \delta_{1} / d r_{1}\right)\right] \\
M_{t}=-D_{1}\left[\left(1 / r_{1}\right)\left(d \delta_{1} / d r_{1}\right)+v_{1}\left(d^{2} \delta_{1} / d r_{1}^{2}\right)\right] \\
D_{1}=E_{1} h^{3} / 12\left(1-v_{1}^{2}\right)
\end{gathered}
$$

where: $D_{1}$ - bending stiffness; $v_{1}$ and $E_{1}$ - Poisson's ratio and Young's elastic modulus of the thin-walled component; $h$ - thickness of the thin wall component.

Substituting,

$$
d^{3} \delta_{1} / d r_{1}^{3}+\left(1 / r_{1}\right)\left(d^{2} \delta_{1} / d r_{1}^{2}\right)+\left[\left(v_{1}-1\right) / r_{1}^{2}\right]\left(d \delta_{1} / d r_{1}\right)=Q / D_{1}
$$

In this case, $P=2 \pi r_{1} Q$ is obtained by lateral force equilibrium. Substituting and simplifying,

$$
d\left[d\left(r_{1} d \delta_{1} / d r_{1}\right) / r_{1} d r_{1}\right] / d r_{1}=P / 2 \pi r_{1} D_{1}
$$

The general solution of this equation yields to the following form

$$
\delta_{1}=P \cdot r_{1}^{2}\left(\ln r_{1}-1\right) / 8 \pi D_{1}+C_{1} r_{1}^{2} / 4+C_{2} \log r_{1}+C_{3}
$$

Due to the clamped edges, the deflection $\left(\delta_{1}\right)$ is zero at the edge of the thin wall $\left(r_{1}=R_{1}\right)$, and the slope of the deflection $\left(d \delta_{1} / d r_{1}\right)$ must be zero for the centre $\left(r_{1}=0\right)$ and edge $\left(r_{1}=R_{1}\right)$.

$$
\begin{gathered}
\delta_{1}=0, \text { for } r_{1}=R_{1} \\
d \delta_{1} / d r_{1}=0, \text { for } r_{1}=0 \text { and } r_{1}=R_{1}
\end{gathered}
$$

where $R_{1}$ - radius of the circular thin wall.

Substituting equation (12) for $\delta_{1}$, the constants of integration $C_{1}, C_{2}$, and $C_{3}$ are obtained

$$
\begin{gathered}
C_{1}=-P\left(\ln R_{1}-1 / 2\right) / 2 \pi D_{1} \\
C_{2}=0 \\
C_{3}=P \cdot R_{1}^{2} / 16 \pi D_{1}
\end{gathered}
$$


Then, the deflection $\delta_{1}$ in the case of symmetric loading is derived by

$$
\delta_{1}=P \cdot R_{1}^{2}\left[1-r_{1}^{2} / R_{1}^{2}+2 r_{1}^{2} \ln \left(r_{1} / R_{1}\right) / R_{1}^{2}\right] / 16 \pi D_{1}
$$

(ii) Asymmetric loading. When the grinding position moves to an eccentric point, for example, a distance of $b$ relative to the thin wall centre $O$, the thin wall is divided into two regions by the cylindrical section of radius $b$. Compared with the symmetric case, the thin wall deflection expression here should contain the term of the azimuth angle $(\theta)$ so as to be varied along the circumferential section. Therefore, we have[6,32]

$$
\delta_{1}\left(r_{1}, \theta\right)=\left\{\begin{array}{l}
\delta_{1 i}\left(r_{1}, \theta\right)=\widehat{K}_{0}+\sum_{m=1}^{\infty} \widehat{K}_{m} \cos m \theta, \text { for } 0<r_{1} \leq b \\
\delta_{1 o}\left(r_{1}, \theta\right)=K_{0}+\sum_{m=1}^{\infty} K_{m} \cos m \theta, \text { for } b \leq r_{1} \leq R_{1}
\end{array}\right.
$$

where

$$
\left\{\begin{array}{l}
K_{0}=a_{0}+b_{0} r_{1}^{2}+c_{0} \log r_{1}+d_{0} r_{1}^{2} \log r_{1} \\
K_{1}=a_{1} r_{1}+b_{1} r_{1}^{3}+c_{1} r_{1}^{-1}+d_{1} r_{1} \log r_{1} \\
K_{m}=a_{m} r_{1}^{m}+b_{m} r_{1}^{m+2}+c_{m} r_{1}^{-m}+d_{m} r_{1}^{-m+2}, m=2,3,4, \ldots
\end{array}\right.
$$

These symbols of $\widehat{K}_{0}, \widehat{K}_{1}, \widehat{K}_{m}$ in deflection function $\delta_{1 i}\left(r_{1}, \theta\right)$ for the inner region can be written in similar forms. The finite requirement at the centre, the boundary conditions, the deformation and shear forces continuity conditions along the loading circle require to act as requirements, and these requirements can be expressed

$$
\begin{gathered}
\hat{c}_{m}=\hat{d}_{m}=0, m=1,2,3,4, \ldots \\
\delta_{1 o}=\partial \delta_{1 o} / \partial r_{1}=0, \text { for } r_{1}=R_{1} \\
\delta_{1 o}=\delta_{1 i}, \partial \delta_{1 o} / \partial r_{1}=\partial \delta_{1 i} / \partial r_{1}, \partial^{2} \delta_{1 o} / \partial r_{1}^{2}=\partial^{2} \delta_{1 i} / \partial r_{1}^{2}, \text { for } r_{1}=b \\
D_{1} \partial\left(\Delta \delta_{1 o}\right)_{r_{1}=b} / \partial r_{1}-D_{1} \partial\left(\Delta \delta_{1 i}\right)_{r_{1}=b} / \partial r_{1}=P\left(1 / 2+\sum_{m=1}^{\infty} \cos m \theta\right) / \pi b
\end{gathered}
$$

From the above conditions, the functions $A_{m}$ and $\hat{A}_{m}$ can be

$$
\left\{\begin{array}{l}
K_{0}=\left[\left(r_{1}^{2}+b^{2}\right) \log \left(r_{1} / R_{1}\right)+\left(R_{1}^{2}+b^{2}\right)\left(R_{1}^{2}-r_{1}^{2}\right) / 2 R_{1}^{2}\right] P / 8 \pi D_{1} \\
K_{1}=-\left[1 / r_{1}+2\left(R_{1}^{2}-b^{2}\right) r_{1} / R_{1}^{2} b^{2}-\left(2 R_{1}^{2}-b^{2}\right) r_{1}^{3} / R_{1}^{4} b^{2}\right. \\
\left.-\left(4 r_{1} / b^{2}\right) \log \left(R_{1} / r_{1}\right)\right] P b^{3} / 16 \pi D_{1} \\
K_{m}=\left\{\left[(m-1) b^{2}-m R_{1}^{2}+(m-1) r_{1}^{2}\right.\right. \\
\left.-m(m-1) b^{2} r_{1}^{2} /(m+1) R_{1}^{2}\right] r_{1}^{m} / R_{1}^{2 m} \\
\left.+\left[r_{1}^{2}-(m-1) b^{2} /(m+1)\right] / r_{1}^{m}\right\} P b^{m} / 8 m(m-1) \pi D_{1}
\end{array}\right.
$$




$$
\left\{\begin{array}{l}
\widehat{K}_{0}=\left[\left(r_{1}^{2}+b^{2}\right) \log \left(b / R_{1}\right)+\left(R_{1}^{2}+r_{1}^{2}\right)\left(R_{1}^{2}-b^{2}\right) / 2 R_{1}^{2}\right] P / 8 \pi D_{1} \\
\widehat{K}_{1}=-\left[2\left(R_{1}^{2}-b^{2}\right) r_{1} / R_{1}^{2} b^{2}+\left(R_{1}^{2}-b^{2}\right)^{2} r_{1}^{3} / R_{1}^{4} b^{4}\right. \\
\left.-\left(4 r_{1} / b^{2}\right) \log \left(R_{1} / b\right)\right] P b^{3} / 16 \pi D_{1} \\
\widehat{K}_{m}=\left\{\left[(m-1) b^{2}-m R_{1}^{2}+R_{1}^{2 m} / b^{2 m-2}\right] r_{1}^{m} / R_{1}^{2 m}\right. \\
+(m-1)\left[1-m b^{2} /(m+1) R_{1}^{2}-R_{1}^{2 m} /(m+1) b^{2 m}\right] \\
\left.r_{1}^{m+2} / R_{1}^{2 m}\right\} P b^{m} / 8 m(m-1) \pi D_{1}
\end{array}\right.
$$

\subsubsection{The function linking tool deformation and pressure}

Assuming the pressure is distributed centro-symmetrically, since the tool deformation of each element is affected by not only the pressure of the located one but also pressure from other elements, the equation of $\delta_{2}=f_{2}(P)$ can be expressed as [33]

$$
\delta_{2}(r)=\left[\left(1-v_{2}^{2}\right) / \pi E_{2}\right] \int_{0}^{2 \pi}\left(q_{i} / 2 \pi l\right) d \varphi_{2}
$$

where $v_{2}$ and $E_{2}$ - Poisson's ratio and Young's elastic modulus of the tool; $l$ and $\varphi_{2}-$ distance and the azimuth angle between the calculated point and the pressure location, respectively (see Fig. 3).

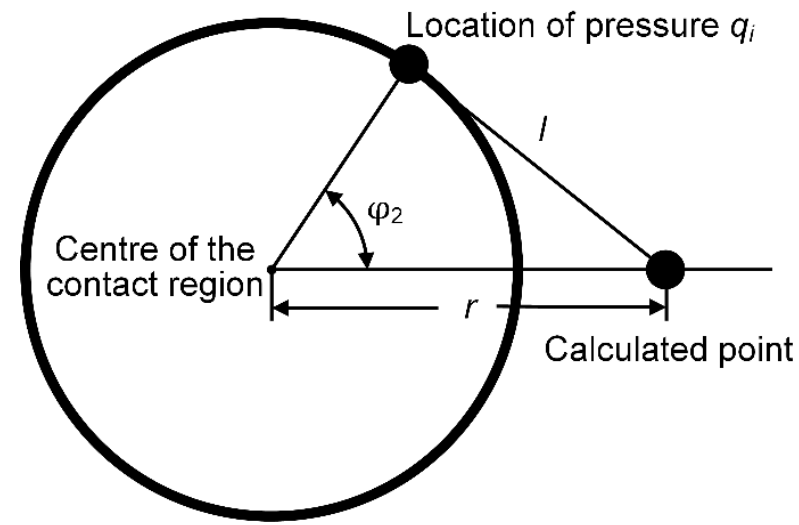

Fig. 3. The position relationship between the calculated point and pressure location [33]

\subsubsection{Solving method of the geometric equilibrium equation}

With the functions linking the deflections of the part and tool with the pressure being defined (Eqs. (18), (19) and (27)), the next step is to solve the pressure between two soft bodies based on the geometric equilibrium equation (Eq. (2)).

The Eq. (2) concerning element centroids is established, then the matrix form of Eq. (2) is

$$
\begin{gathered}
A_{j i} q_{i}=B_{j}, i=1,2,3, \ldots n, j=1,2,3, \ldots n \\
B_{j}=e-s_{0 j}
\end{gathered}
$$

where: $A_{j i}$ - similar stiffness matrix from Eqs. (18), (19) and (27); $B_{j}$ - compliant motion 
matrix (at $j$ th position); $i$ th - the sequence of the calculated pressure; and $j$ th - the sequence of chosen field points (i.e. centroids and nodes of discretized elements); $s_{0 j}$-initial distance for the chosen field points from Eq. (3).

However, the ill-posed nature of Eqs. (28) and (29) may introduce physically meaningless results if they are solved directly. Hence, to decrease this possibility and a violent oscillation of contact pressure, we use the Method of Redundant Field Points (RFP) and the Functional Regularization (FR) [34-36] to calculate contact pressure distributed on each element ( $n$ unknowns)

The contact pressure is obtained from the solution of Eq. (28) (details are shown in Appendix B) after the contact area ( $a$ calculated from Eq. (A.5) [37]) is updated for the positive pressure values. Then, the thin wall deflection $\left(\delta_{1}\right)$ and the actual tool offset $\left(\delta_{2}\right)$ used for the tool deformation are calculated from Eqs. (2), (18) and (19) under a nominal tool offset $(e)$.

\subsection{Implications of part/tool deformations under pressure in shape adaptive grinding (SAG)}

Having the relationship between the given and actual tool offsets (Eqs. (2), (18) and (19)), some specific influences on the SAG process can be highlighted: the maximum acceptable deformation of thin walls in elastic region, and the influence function of relative positions between the tool and workpiece. These will be used as design requirements of nominal tool offsets.

\subsubsection{Elastic domain determination of the thin wall component}

The designed tool offset enables not only the uniformity of material removal rate but also the elastic domain of the thin wall deflection. The stress of the thin wall is designed to be lower than its elastic limit as the component plastic deformation is not acceptable in applications. The bending moments of thin walls for symmetrical loads can be expressed as [32]

$$
\left\{\begin{array}{l}
M_{r}\left(r_{1}\right)=-P\left[1+\left(1+v_{1}\right) \log \left(r_{1} / R_{1}\right)\right] / 4 \pi \\
M_{t}\left(r_{1}\right)=-P\left[v_{1}+\left(1+v_{1}\right) \log \left(r_{1} / R_{1}\right)\right] / 4 \pi
\end{array}\right.
$$

For asymmetrical loads,

$$
\left\{\begin{array}{l}
M_{r}\left(r_{1}, \theta\right)=-D_{1}\left[\partial^{2} \delta_{1} / \partial r_{1}^{2}+v_{1}\left(\partial \delta_{1} / r_{1} \partial r_{1}+\partial^{2} \delta_{1} / r_{1}^{2} \partial \theta^{2}\right)\right] \\
M_{t}\left(r_{1}, \theta\right)=-D_{1}\left(v_{1} \partial^{2} \delta_{1} / \partial r_{1}^{2}+\partial \delta_{1} / r_{1} \partial r_{1}+\partial^{2} \delta_{1} / r_{1}^{2} \partial \theta^{2}\right)
\end{array}\right.
$$

where $\partial \delta_{1} / \partial r_{1}, \partial^{2} \delta_{1} / \partial r_{1}^{2}$, and $\partial^{2} \delta_{1} / \partial \theta^{2}$ are expressed in Appendix C.

Then stresses on thin walls should respect the condition 


$$
\left\{\begin{array}{l}
\sigma_{r}\left(r_{1}, \theta\right)=-6 D_{1} M_{r} / h^{2}<\sigma_{e} \\
\sigma_{t}\left(r_{1}, \theta\right)=-6 D_{1} M_{t} / h^{2}<\sigma_{e}
\end{array}\right.
$$

where $\sigma_{r}$ and $\sigma_{t}$ - radial and circumferential stresses, respectively; $\sigma_{e}$ - yield strength.

\subsubsection{Controlling the tool offset as a function of the relative position between the grinding tool and thin wall}

The tool moves from the initial contact without pressure (initial positions filled by light blue) with a distance of $e_{2}$ at $\mathrm{P} 2$ relative to the thin wall (a), leading to the thin wall deflection being unequal $\left(\delta_{1-2-2} \neq \delta_{1-2-1} \neq \delta_{1-2-3} \neq \delta_{1-2-4} \neq \delta_{1-2-5}\right)$ between the contact (P2) and noncontact positions (P1 and P3-5). When the relative positions between the tool and thin wall are changed under the same nominal tool offset (b), the thin wall deflections at corresponding contact positions are different along with the radial directions $\left(\delta_{1-1-1} \neq\right.$ $\delta_{1-2-2} \neq \delta_{1-3-3}, \delta_{1-1-1}=\delta_{5-5-5}$ and $\left.\delta_{2-2-2}=\delta_{4-4-4}\right)$. The thin wall deflection at grinding positions is (when $r_{1}=b$ from Eqs. (18) (19) (25) and (26))

$$
\begin{gathered}
\delta_{1}(b, \theta)=\left\{\begin{array}{l}
P R_{1}^{2}\left[1-b^{2} / R_{1}^{2}+2 b^{2} \ln \left(b / R_{1}\right) / R_{1}^{2}\right] / 16 \pi D_{1}, b \rightarrow 0 \\
K_{0}+\sum_{m=1}^{\infty} K_{m} \cos m \theta, b>0
\end{array}\right. \\
\left\{\begin{array}{l}
K_{0}=\left[4 b^{2} \log \left(b / R_{1}\right)+R_{1}^{2}-b^{4} / R_{1}^{2}\right] P / 16 \pi D_{1} \\
K_{1}=-\left[3-4 b^{2} / R_{1}^{2}+b^{4} / R_{1}^{4}-4 \log \left(R_{1} / b\right)\right] P b^{2} / 16 \pi D_{1} \\
K_{m}=\left\{\left[2(m-1) b^{2}-m R_{1}^{2}\right] b^{m} / R_{1}^{2 m}+2 /(m+1) b^{m-2}\right. \\
\left.-m(m-1) b^{m+4} /(m+1) R_{1}^{2 m+2}\right\} P b^{m} / 8 m(m-1) \pi D_{1}
\end{array}\right.
\end{gathered}
$$

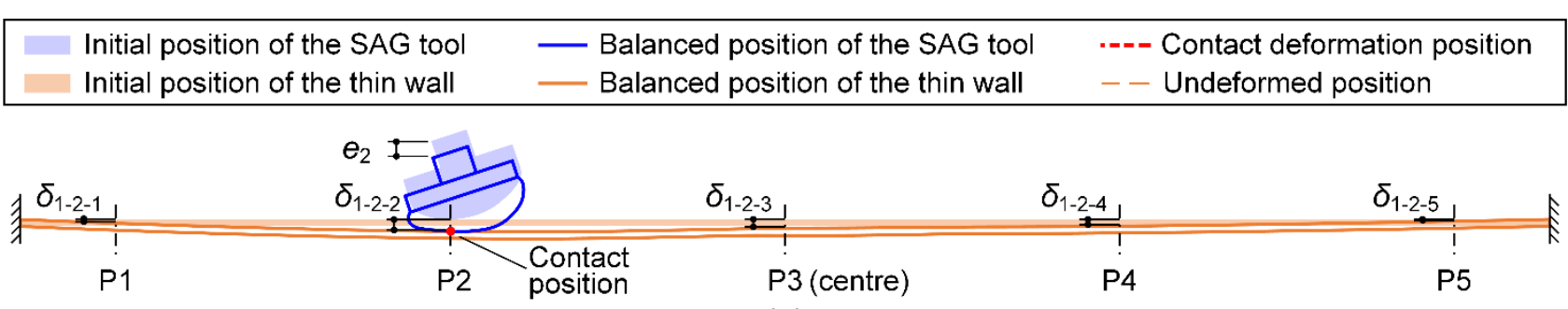

(a)

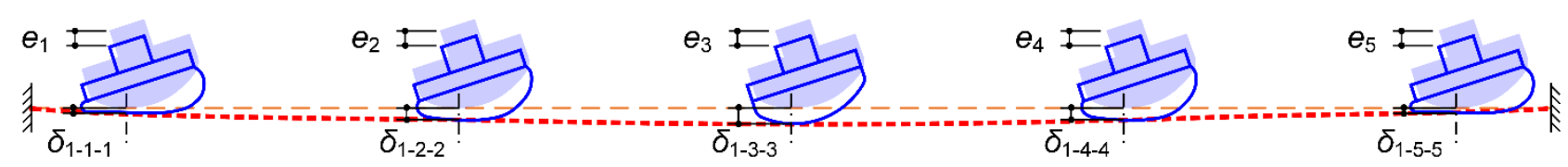

(b)

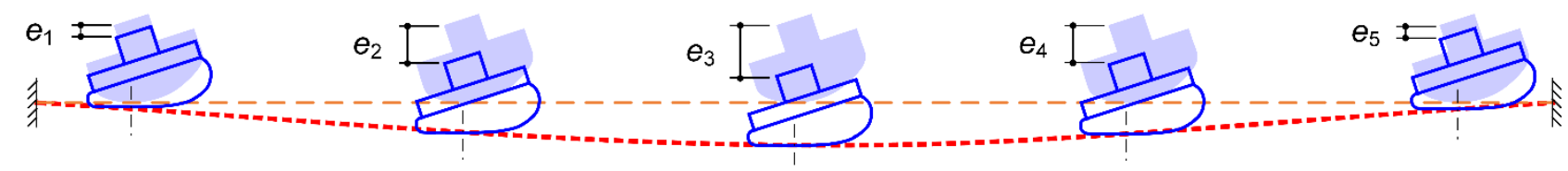

(c)

Fig. 4. The influences of the relative positions between the grinding tool and the thin wall on thin wall deflections and tool deformations. The thin wall deflects differently at different positions (a), and the thin-wall deflections at 
the contact positions are varied as well as the tool deformations (b). The proposed strategy is to input the timevariant tool offsets so as to obtain the same tool deformation (c).

In the grinding process, the second aspect (the thin wall deflection at contact positions in Fig. $4 \mathrm{~b}$ ) is of interest as material removal occurs in the contact area, and it is more dependent on the deflection features of the thin wall rather than the tool, meaning, the dissimilarity in thin wall deflection with relative positions between the grinding tool and the thin wall (i.e. grinding positions). Considering the symmetry in shapes and boundary conditions of thin walls for the studied case, the deflections are equal (i.e. $\delta_{1-1-1}=\delta_{5-5-5}, \delta_{2-2-2}=\delta_{4-4-4}$ ) when the grinding positions are radially symmetric (P1 and P5, P2 and P4 in Fig. 4a).

Conventional strategies for compliant grinding processes accept the nominal tool offset with a constant value (see $e_{1}=e_{2}=e_{3}=e_{4}=e_{5}$ in Fig. 4b). However, the tool is increasingly deformed with the variation of grinding positions (see the tool shapes in solid blue lines in Fig. $4 \mathrm{~b})$, which indicates an increased normal contact load. It is because the thin wall deflection variation with grinding positions relies on both the position-dependent stiffness and changing normal contact loads although the tool stiffness is not relevant to the relative positions between two.

For the case chosen here, i.e. the circular shape of thin walls with fully clamped edges, the deflection at the centre is more obvious than the one near the edges. That is, the nominal tool offset is mostly used for deflecting the thin wall when the tool is at the thin wall centre but deforming the tool when the tool is near the thin wall edges. Such being the case, the tool should be given a bigger offset at the centre so as to obtain the desired material removal rate under a bigger actual tool offset used for its deformation. In other words, the nominal tool offsets should be variant with the relative positions between the tool and the thin wall (see in Fig. $4 \mathrm{c}, e_{1} \neq e_{2} \neq e_{3} \neq e_{4} \neq e_{5}$ ) with the aim of achieving an equalized actual tool offset (see the tool shapes in solid blue lines in Fig. 4c).

Based on the above calculations (sections 2.1 and 2.2), the control strategy is shown in Fig. 5. Under the desired MRR, the actual tool offset used for tool deformations is obtained by calibration tests applying on the rigid block (which will be introduced in section 3.2). The radius of the candidate contact region in section 2.1 was initially given as the Eq. (A.4) in Appendix A to start the calculation, then replaced with Eq. (A.5) after the tool deformation was obtained from section 2.2. After the nominal tool offsets were designed, owing to the sub-aperture nature of SAG, the control strategy should contain the check of grinding positions relative to the workpiece in order to cover the whole desired grinding area. 


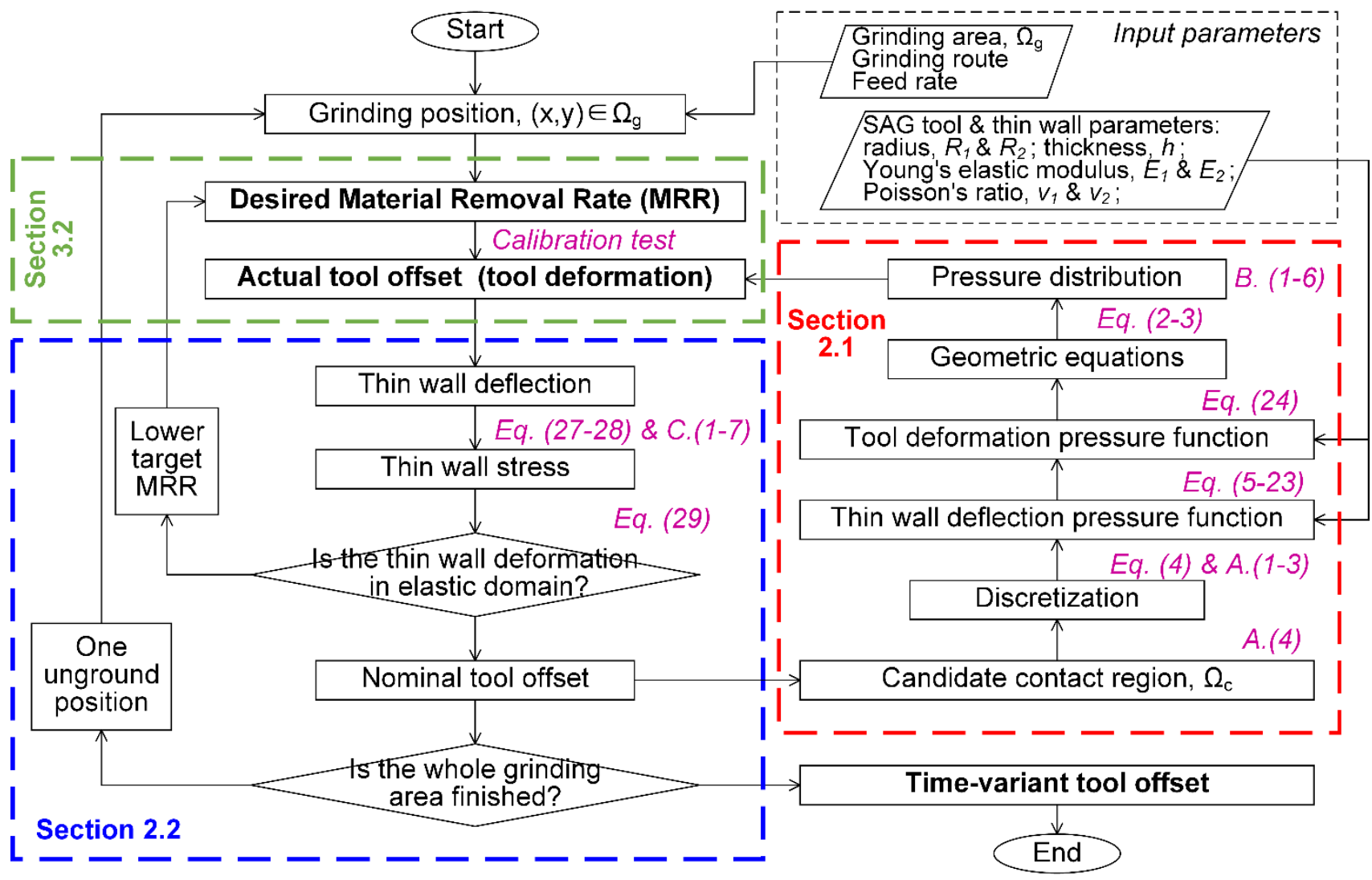

Fig. 5. Flowchart of the control strategy for compliant grinding of low stiffness components

\section{Experimental methodology and model calibration}

\subsection{Experimental methodology}

To validate the proposed control strategy, a thin wall of brushed aluminium (Type: 1060) with an in-plane dimension to thickness ratio of 160 (diameter: $80 \mathrm{~mm}$, thickness: $0.5 \mathrm{~mm}$ ) was used as the low-rigidity component in this study (as shown in Table 1). The thin wall is actually square with a dimension of $125 \mathrm{~mm}$, but its effective shape is circular with a diameter of $80 \mathrm{~mm}$ after the clamping. It was fully clamped along its edge by two engineered ring clamps and the ALUFIX Modular Fixturing System while no other support was provided below the thin wall except for the boundary support (see in Fig. 6). The used thin wall parts were chosen as flat as possible (flatness $<20 \mu \mathrm{m}$ ). The Young's modulus and Poisson ratio of aluminium thin walls are 70Gpa and 0.3 , respectively, as shown in its material specifications. The shape adaptive grinding tool with a radius of $12 \mathrm{~mm}$ was used as the compliant tool whose Young's elastic modulus can be fitted as a cubic polynomial function of the tool hardness [38].

Grinding tests have been carried out on a 5-axis CNC machine (Hurco VMX42SRTi). A Kistler 9255B dynamometer was used to acquire the normal grinding forces. The surface profile measurement of the thin wall was employed on a Coordinate Measuring Machine (Hexagon 
Metrology) with a resolution of $0.01 \mu \mathrm{m}$ before and after grinding so as to validate the elastic deformation and removal depth of the thin walls.

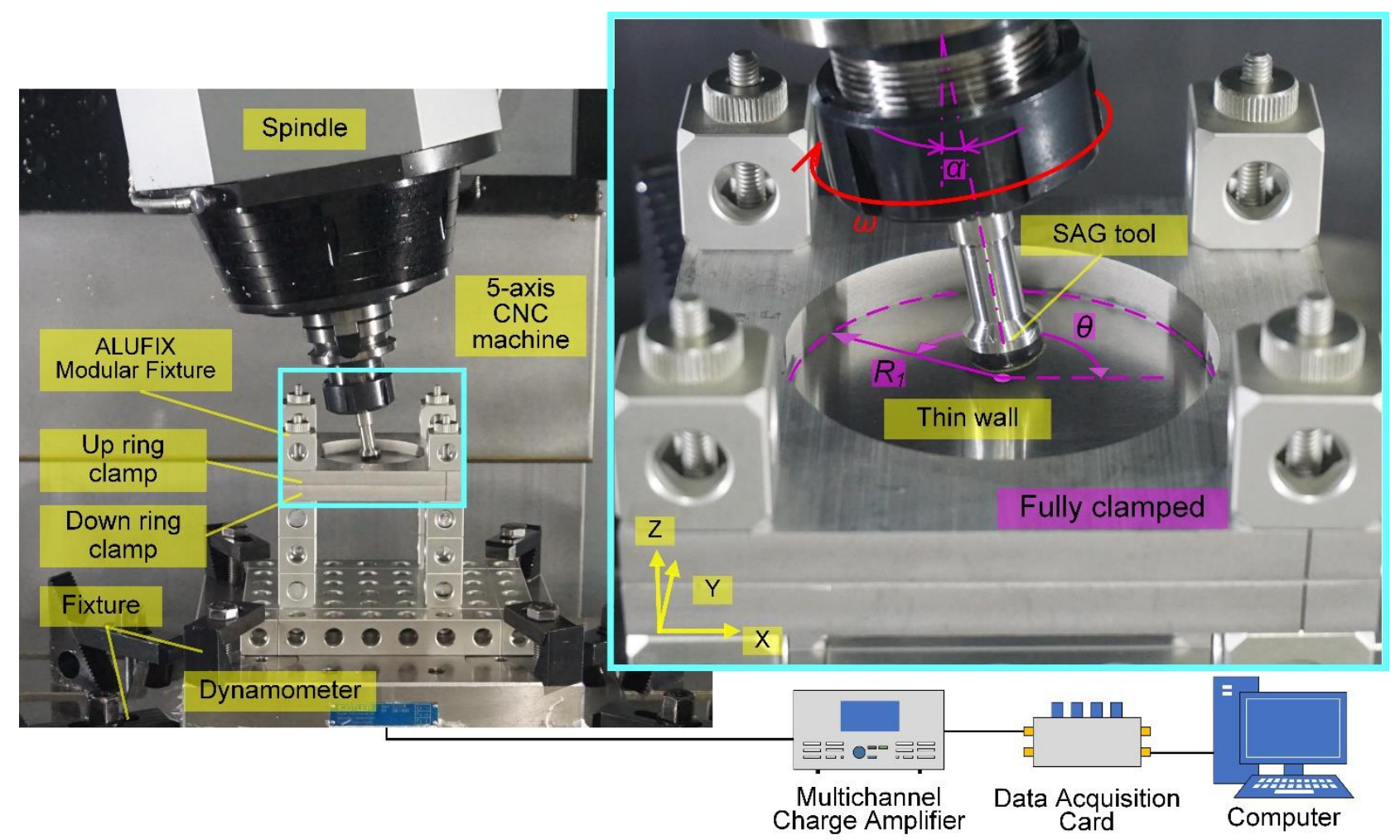

Fig. 6. Experimental setups of continuous and spot grinding of thin-wall components with the SAG tool.

The grinding spot tests for the influence function calibration were conducted on a thick aluminium block with the SAG tool firstly, using processing parameters shown in Table 1. The ground surface in the spot size was scanned by a 3D surface measurement system (Alicona) for material removal calibration under the actual tool offset. Then a series spot grinding tests (grinding tests without a feed rate, using the same processing parameters shown in Table 1) were performed over different radial positions of the circular aluminium thin-wall part, which is aimed at verifying the thin wall deflection calculated from our contact model (see Fig. 6). Last, in a whole circle area of the thin wall part, continuous grinding (with feed rate) test results were compared between the designed tool offset from our controlling process model and the constant tool offset from the conventional method.

Table 1 Parameters of shape adaptive grinding experiments.

\begin{tabular}{llll}
\hline Aluminium thin wall & & \multicolumn{2}{l}{ Main processing parameters } \\
\hline Dimension: & $125^{\star} 125^{\star} 0.5 \mathrm{~mm}$ & Nominal tool offset & $0.1 \sim 0.5 \mathrm{~mm}$ \\
Effective dimension: & $\Phi 80^{*} 0.5 \mathrm{~mm}$ & Grinding position & $0.0,10.0,20.0 \mathrm{~mm}$ \\
Young's elastic modulus & $70 \mathrm{Gpa}$ & relative to the centre &
\end{tabular}




\begin{tabular}{llll} 
Poisson's Ratio: & 0.3 & $\begin{array}{l}\text { Grinding area } \\
\text { Grinding path }\end{array}$ & $\begin{array}{l}\text { Circle }(\$ 50 \mathrm{~mm}) \\
\text { Raster }\end{array}$ \\
& & Track spacing & $0.2 \mathrm{~mm}$ \\
\cline { 1 - 1 } SAG tool & $12 \mathrm{~mm}$ & Feed rate & $0.0,100 \mathrm{~mm} / \mathrm{min}$ \\
\hline Radius: & $40 \mu \mathrm{m}$ & Dwell time & $20 \mathrm{~s}$ \\
Grain size: & $85 \mathrm{~A}$ & Spindle speed & $500 \mathrm{rpm}$ \\
Hardness: & & Precess angle & $10^{\circ}$ \\
& & &
\end{tabular}

\subsection{Calibrating the SAG process}

The relation between the tool deformation and material removal rate was obtained by applying the SAG tool on a thick aluminium block (length: $100 \mathrm{~mm}$, width: $50 \mathrm{~mm}$, height: $30 \mathrm{~mm}$ ) using process conditions in Fig. 6 identical to those described in section 3.1. Over non-overlapping positions of the aluminium block, a set of spot grinding tests were given different tool offsets from $0.1 \mathrm{~mm}$ to $0.4 \mathrm{~mm}$ with an interval of $0.05 \mathrm{~mm}$. Here, the aluminium block is considered as the rigid component without macro deformation (i.e. bending). Then the nominal tool offset of the SAG tool can be regarded as the actual tool offset, that is, the tool offsetting motion is fully used to deform the compliant tool. Material removal rate (see red line in Fig. 7) and normal grinding force (see blue line in Fig. 7) show the same increasing trend with the actual tool offset, as known from Preston's law that material removal is in direct proportion to contact pressure. The increasing trend can be fitted to be linear due to the combined effects of spot area and contact pressure because they both follow a square root law of the actual tool offset [37]. The rubber material gradually adapts to the surface after being removed, so the normal grinding force decreases with dwell time (see the left top figure in Fig.7). In grinding processes, the dwell time of compliant tools relies on the feed rate $(0.0 \sim 1000 \mathrm{~mm} / \mathrm{min})$, to better validate contact loads, the average normal grinding forces during the first 3 seconds of dwell time are chose to compare with actual tool offsets. The material removal rate is equal to the integrated removal volume divided by the dwell time. As the material removal rate is not influenced by the dwell time [25], the material removal rate calculated from the spot grinding tests can be used in continuous cases. 


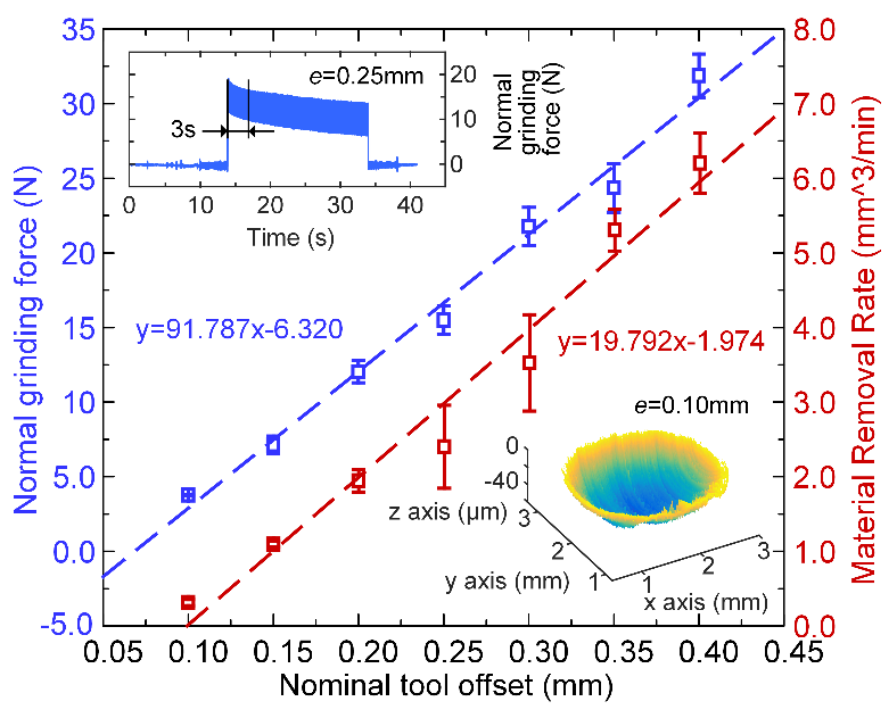

Fig. 7. Normal grinding force and material removal rate versus nominal tool offset (tool deformation) on the aluminium block with the SAG tool in spot grinding tests.

\section{Model validation and discussion}

\subsection{Spot grinding tests}

After the actual tool offset is computed by the contact deformation model under a nominal tool offset, the actual material removal rate in the compliant grinding of thin walls can be calculated based on interpolation from the data shown in Fig. 7 (see purple rectangles in Fig. 8a).

The calculated material removal rates show a good agreement with the measured one, indicating the material of circular aluminium thin walls is removed less than the removal of the rigid aluminium block with the SAG tool due to the thin wall deflection (compare the material removal rate in Figs. 7 and 8 when the tool offset is $0.3 \mathrm{~mm}$ ). With the increase of the distances between the circular thin wall centre and grinding positions, the material removal is increased (see the increasing trend in Fig. 8 with the grinding positions from 0 to $20 \mathrm{~mm}$ ) but still less than the aluminium block case. It means the thin wall deflection is decreased when the tool moves from the centre to the edges of the thin wall, which is consistent with the proposed model. As expressed in Eqs. (33) and (34), with the increase of $b$, the thin wall deflection $\left(\delta_{1}\right)$ is nonlinearly decreased, and this is the combined actions of the grinding positions (increased) and normal contact loads (increased, see in Fig. 8b) under the same nominal tool offset. 


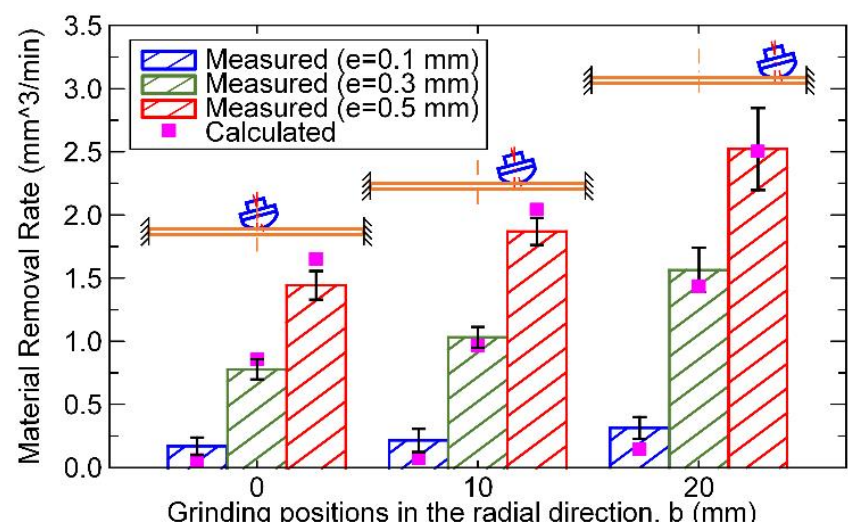

(a)

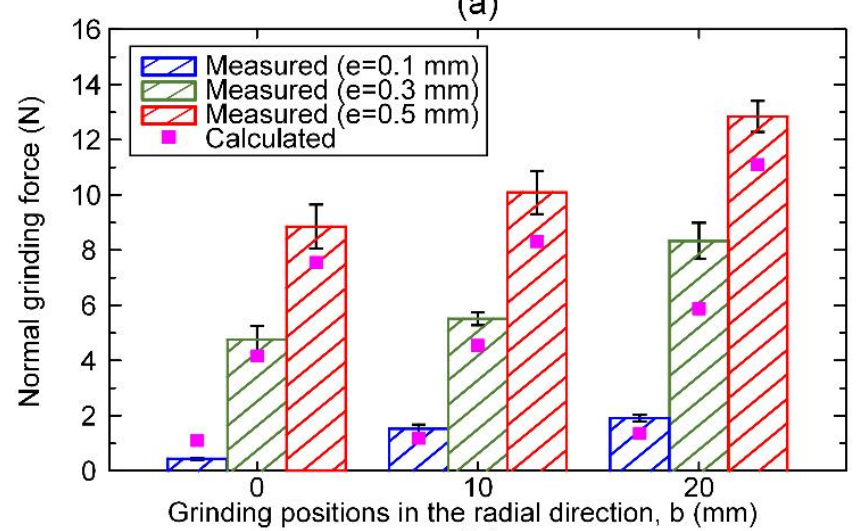

(b)

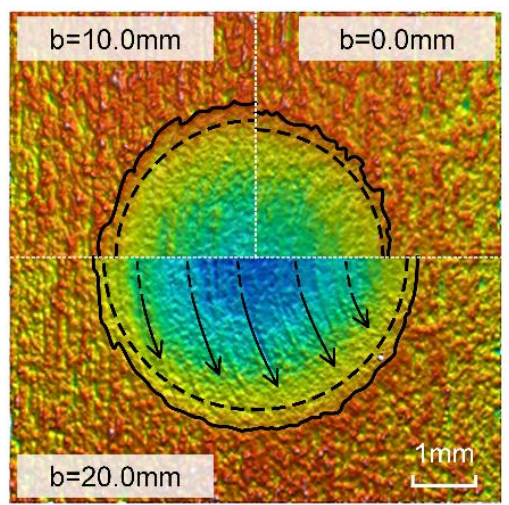

(c)

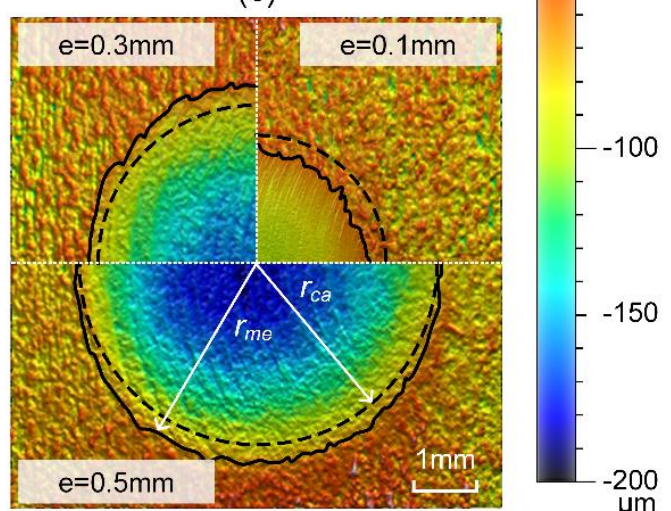

(d)

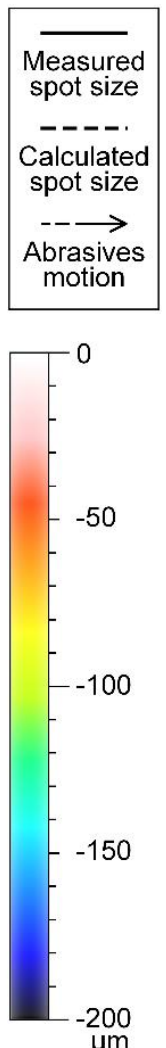

Fig. 8. Material removal of circular aluminium thin walls in spot grinding with SAG tools. The material removal rate (a) and normal grinding force (b) show the increasing trends with grinding positions in the radial direction as well as nominal tool offsets. The spot sizes in area and depth reflect the tool deformation with the same trend as the material removal rate (c and $d$ ).

This change also reflects on the spot sizes (see Fig. 8c), that is, the spot size increases when the SAG tool moves from the centre to the edge of the thin wall. The areas of thin walls near the clamped edges have a higher stiffness than the centre, leading to a higher actual tool offset used for tool deformation, and higher compression loads. For a compliant sphere, higher loadings have effects not only on the deformation depth (material removal depth) but also the contact area (spot size). At the same grinding position, with the nominal tool offsets increasing, the actual tool offsets increase as well as the spot size (see Fig. 8d), which is in line with the depiction of Eq. (A.5) in Appendix A.

In Fig. $8 \mathrm{c}$ and $\mathrm{d}$, the maximum error $\left(\left|r_{m e}-r_{c a}\right| / r_{m e} \times 100 \%\right)$ between the measured and calculated spot sizes is $13 \%$. It is found that the bigger error most likely occurs near the edges where abrasive motion starts or ends (see the top or the bottom edges of the spot in Fig. 8c and d) and in the circumstance of lower tool offsets (see the right top figure in Fig. 8d). In the compression deformation process, the SAG tool surfaces above the contact zone are slightly expanded. The extruded abrasives bonded in pellets start to interact with the workpiece 
surface thanks to the tool spindle rotation, lengthening the distance of spot size along the abrasive motion direction, instead of a perfect circle spot as we assumed. The elastic modulus of the tool relies on all components. The more rigid fabric and abrasives may present a major role when a little deformation occurs. It means the actual equivalent elastic modulus of the tool is a little bit higher than the assumed one only from rubber when there is a small deformation.

\subsection{Continuous grinding tests}

The deflection of thin walls in compliant grinding is related to grinding positions even for a centrosymmetric \& axisymmetric thin wall, which indicates that nominal tool offsets should be designed in a time-variant way to obtain a constant material removal rate in grinding a complete surface of thin walls (see in Fig. 9a), as discussed in Section 2.2.2.

Based on the relationship between the material removal rate and the actual tool offset (Section 3.2), the aim is to obtain a constant actual tool offset. This requires a constant contact force that yields the same tool deformation as illustrated by the corresponding relation in the calibration tests (see section 3.2) and validated by the spot grinding tests (see section 4.1). In continuous grinding, the material removal rate could be replaced by the material removal depth as the ground surface evaluation parameter in the same processing conditions (e.g. same spindle speed, feed rate, process angle and grinding routes in table 1). The desired removal depth is the one obtained from the case where the compliant tool is performed on the rigid aluminium block with a constant tool offset (see dot lines in Fig. 9e).

The ground surface of a circular aluminium thin wall under a constant tool offset presents a non-flat profile (i.e. high at the centre but low along edges, see in Fig. 9c), and a lower removal depth than the flat surface obtained from time-variant tool offsets (see in Fig. 9d). To better depict and evaluate their differences, cross-sections along four directions $\left(0^{\circ}, 45^{\circ}, 90^{\circ}\right.$ and $\left.135^{\circ}\right)$ are introduced in Fig. 9e. Under constant tool offsets, the profile fluctuation has an amplitude of around $50 \mu \mathrm{m}$ in the ground area (see dash lines in Fig. 9e), and the surface occurs unexpected deformation leading to the ground surface above the initial (see blue and red dash lines in Fig. 9e). The interactions between the tool and workpiece include out-plane (outside the $x y$ plane) and in-plane (in the $x y$ plane) cases. The out-plane interactions are the normal contact loads which provide pressure for material removal and induce the thinwall deflection. For the same desired removal depth, the normal contact loads under constant tool offsets are small and nonuniform relative to the case of time-variant tool offsets. The inplane interactions derive from the material removal related to the abrasive and tool motion but only occur on the upper surfaces of thin walls. This leads to a stretched upper surface of 
the thin-walled part and an unstretched lower surface. In an exaggerated sense, the stretched upper surface is larger than the unstretched lower surface of the thin-walled part and this will lead to upwards deformation of the thin wall. The normal contact loads under the given tool offset are not enough although they can stop the upward deformation. Hence, it appears that the final position may be higher than the initial surface when the given tool offset is small (see the blue dash line in Fig. 9e). The phenomena are more obvious when the tool offsets are smaller, i.e. the smaller nominal tool offset (compare the green, red and blue dash lines in Fig. 9e). In other words, the non-flat ground surface occurring in the conventional approach is not only from the non-uniform material removal but also the post-processing deformation.

On the contrary, no unexpected deformation occurs in the case of time-variant tool offsets. This is because, the downward normal contact loads play a dominant role in thin-wall deformations although the in-plane forces in the upper and lower surfaces are still unbalanced. The downward deformation from normal contact loads resists the upward unexpected deformation from in-plane forces. For the same desired material removal depth, the normal contact loads in the time-variant case are higher and more uniform than those in the constant tool offset case, so the unexpected deformation is less visible.

The surfaces ground under the time-variant tool offsets reach the desired removal depth with an average error $\leq 10 \%$ and achieve $11.2 \mu \mathrm{m}, 24.2 \mu \mathrm{m}$ and $41.8 \mu \mathrm{m}(\mathrm{P}-\mathrm{V})$ accuracy, respectively (see Table 2). Compared with constant tool offsets used in the conventional approach, the surfaces ground under time-variant tool offsets show a better agreement with the desired removal depth and less profile fluctuation. It is found that the gaps of the timevariant case relative to the desired removal depth tends to be bigger when deeper material is removed (see the green solid and dot lines in Fig. 9e). With a higher nominal tool offset, the normal contact loads are dominant. However, this could induce the maximum stress above the yield strength of chosen material which is not under the consideration of the proposed method. That is, the thin wall components partly reach the plastic domain when the tool is near the thin wall centre, which is not acceptable in machining (see Fig. 10).

Our model is able to obtain the desired constant material removal rate of thin-wall components, by controlling the normal offset of the compliant tool based on the grinding positions in the elastic domain. The radius, grain size, hardness, spindle speed and precess angle of the SAG tools are contributing factors for tool influence functions, and the relation between the given and actual tool offsets is related to the dimensions, boundary conditions and material properties of thin walls and tools. Although only a simple case is presented here, the controlling method is likely to be adapted to other circumstances of precision machining 


\section{between two elastic bodies.}

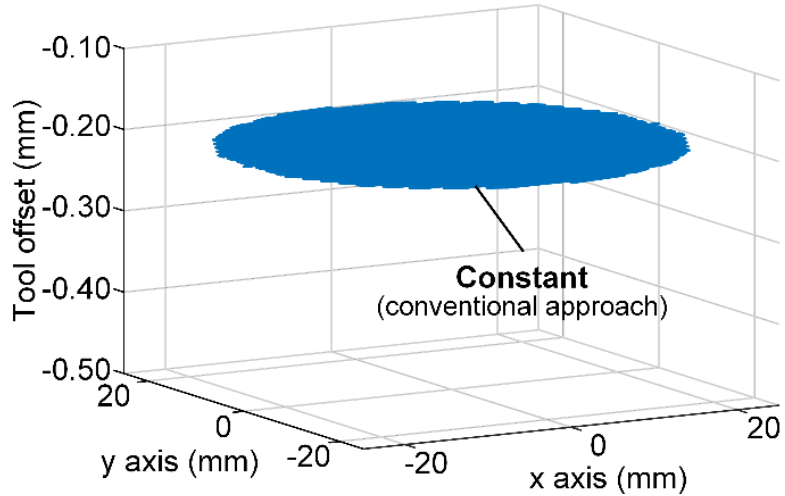

(a)

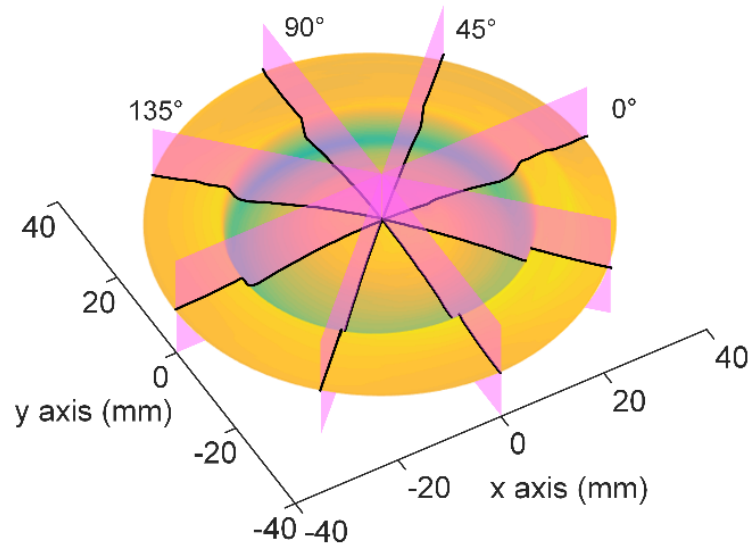

(c)

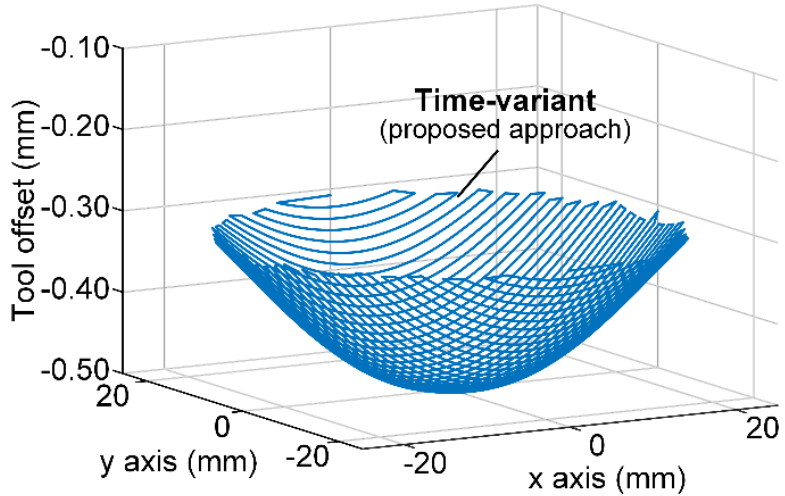

(b)

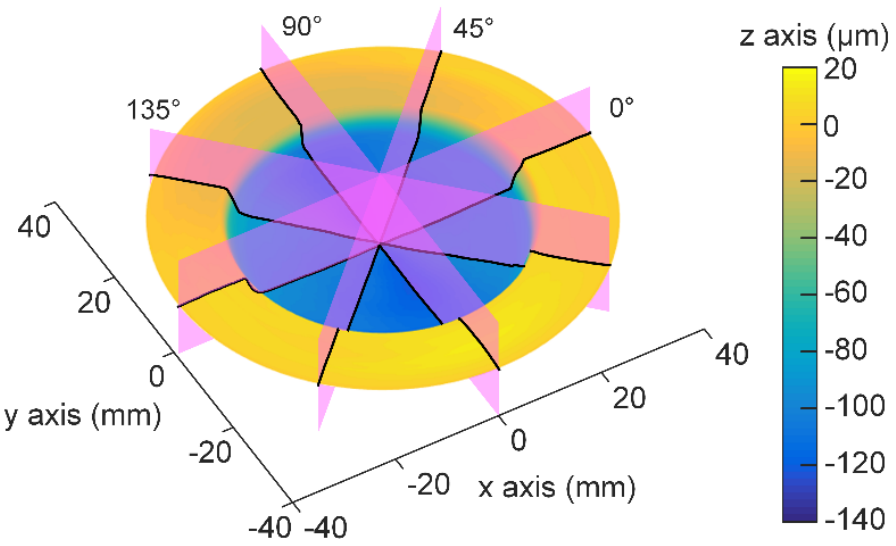

(d)

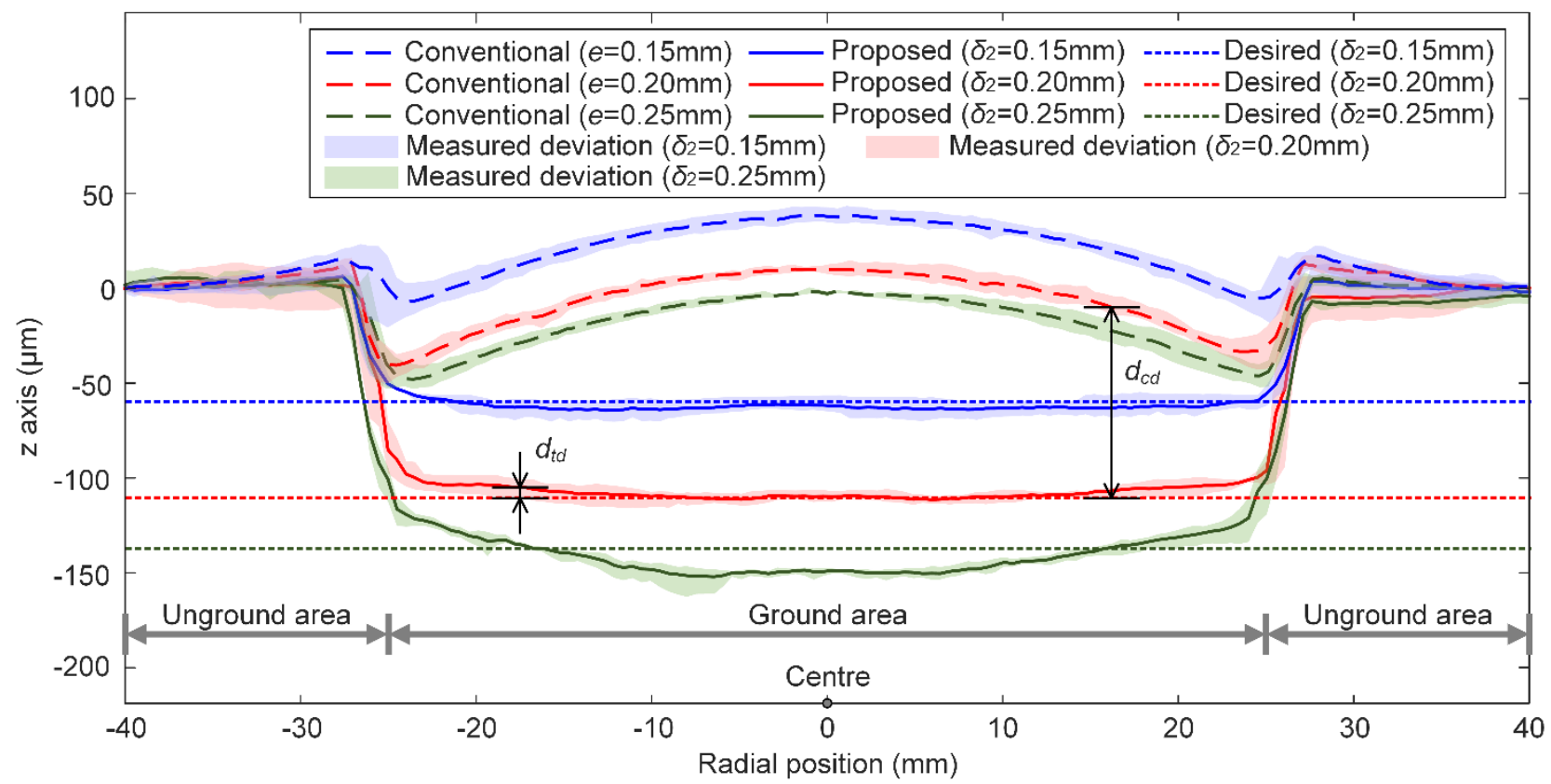

(e)

Fig. 9. Ground surface comparisons between the constant (a) and time-variant tool offsets (b). The material is removed uneven and less in conventional approaches with constant tool offsets (3D view of ground surfaces in c) than time-variant tool offsets (3D view of ground surfaces in d). An unexpected deformation is discovered in the constant case by comparing the cross sections of ground surfaces (e). 
Table 2 Errors between desired and actual removal depths in the cases of conventional and novel approaches.

\begin{tabular}{|c|c|c|c|c|c|c|c|c|c|c|c|}
\hline \multirow{3}{*}{\multicolumn{2}{|c|}{$\begin{array}{l}\text { Errors relative } \\
\text { to the desired } \\
\text { depth }(\mu \mathrm{m})\end{array}$}} & \multicolumn{9}{|c|}{ Radial positions (mm) } & \multirow{3}{*}{$\begin{array}{l}\text { Average } \\
\text { errors } \\
\text { (ground) } \\
(\mu \mathrm{m})\end{array}$} \\
\hline & & \multicolumn{2}{|c|}{ Unground } & \multicolumn{5}{|c|}{ Ground } & \multicolumn{2}{|c|}{ Unground } & \\
\hline & & -40 & 30 & 25 & 12.5 & 0 & 12.5 & 25 & 30 & 40 & \\
\hline \multirow{2}{*}{$\begin{array}{l}\delta_{2}= \\
0.15 \\
\mathrm{~mm}\end{array}$} & Proposed & 60.3 & 64.1 & $\begin{array}{c}9.4 \\
(15.7 \%)\end{array}$ & -4.4 & -1.8 & -2.8 & 4.3 & 61.6 & 57.9 & $\begin{array}{c}0.9 \\
(1.5 \%)\end{array}$ \\
\hline & $\begin{array}{l}\text { Conventi } \\
\text { onal }\end{array}$ & 60.0 & 70.6 & 58.9 & 85.4 & $\begin{array}{c}97.8 \\
(163.2 \%)\end{array}$ & 87.0 & 55.2 & 71.6 & 60.8 & $\begin{array}{c}76.8 \\
(128.2 \%) \\
\end{array}$ \\
\hline \multirow{2}{*}{$\begin{array}{l}\delta_{2}= \\
0.20 \\
\mathrm{~mm}\end{array}$} & Proposed & 111.0 & 113.3 & $\begin{array}{c}25.3 \\
(22.9 \%)\end{array}$ & 2.3 & 1.1 & 1.9 & 14.6 & 105.9 & 111.0 & $\begin{array}{c}9.0 \\
(8.2 \%)\end{array}$ \\
\hline & $\begin{array}{l}\text { Conventi } \\
\text { onal }\end{array}$ & 110.7 & 118.2 & 70.4 & 108.2 & $\begin{array}{c}120.5 \\
(108.8 \%)\end{array}$ & 108.4 & 80.2 & 119.7 & 110.7 & $\begin{array}{c}97.5 \\
(88.1 \%)\end{array}$ \\
\hline \multirow{2}{*}{$\begin{array}{l}\delta_{2}= \\
0.25 \\
\mathrm{~mm}\end{array}$} & Proposed & 139.2 & 140.5 & 35.8 & -7.0 & -11.7 & 5.7 & $\begin{array}{c}36.7 \\
(26.7 \%)\end{array}$ & 129.4 & 133.2 & $\begin{array}{c}9.6 \\
(7.0 \%)\end{array}$ \\
\hline & $\begin{array}{l}\text { Conventi } \\
\text { onal }\end{array}$ & 137.2 & 140.5 & 96.3 & 121.2 & $\begin{array}{c}134.7 \\
(98.0 \%)\end{array}$ & 123.1 & 92.9 & 140.6 & 137.7 & $\begin{array}{c}113.7 \\
(82.7 \%)\end{array}$ \\
\hline
\end{tabular}

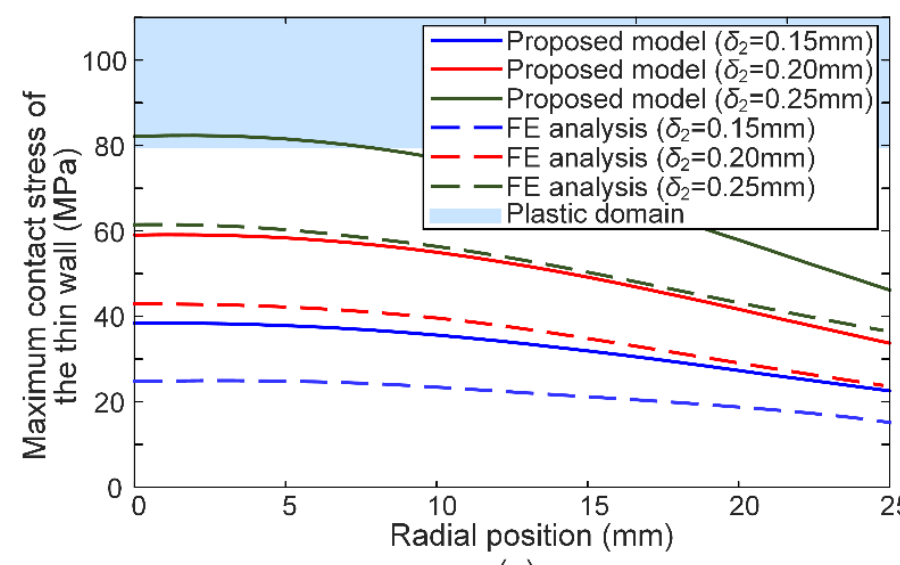

(a)

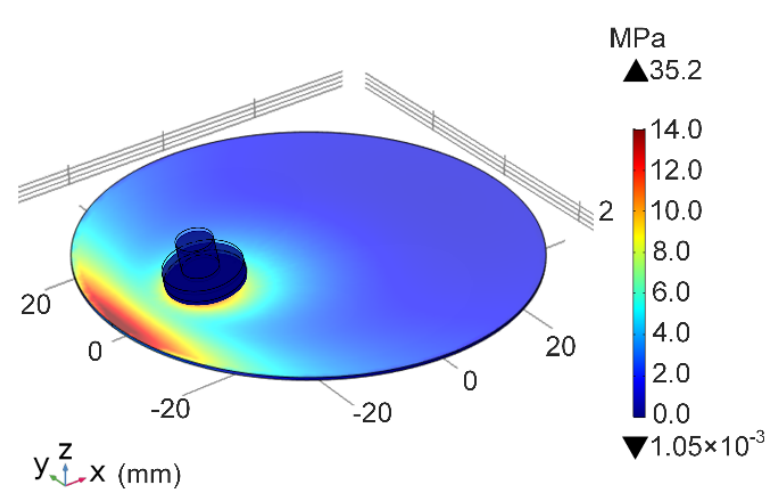

(b)

Fig.10. Maximum contact stresses of thin walls are calculated based on the proposed model and finite element analysis (a) when the complaint tool changes the grinding positions relative to the thin-wall centre (b) under the time-variant tool offsets.

\section{Conclusions}

A control strategy was developed to achieve a constant material removal rate on low-rigidity 
components with compliant tools. The main contributions of this paper are drawn as follows:

- This paper addressed for the first time the contact problem involving dual compliances in machining. The proposed model enables the prediction of the thin wall deflection and tool deformation under a nominal tool offset. It was validated by spot grinding tests employed on thin aluminium walls with SAG tools.

- Further, this paper proposed a new control strategy, i.e. by controlling the nominal tool offset in such a way of being time-variant in grinding thin-walled parts with SAG tools, to obtain a constant material removal rate based on the position-dependent characteristics of the thin wall deflections.

- The surfaces ground under the time-variant tool offsets (proposed approach) reach the desired removal depth with an average error of $\leq 10 \%$ and achieve $11.2 \mu \mathrm{m} \sim 24.2 \mu \mathrm{m}(\mathrm{P}-\mathrm{V})$ accuracy in the elastic domain, compared with the error of $76.8 \% \sim 113.7 \%$ and accuracy of $42.6 \mu \mathrm{m} \sim 50.1 \mu \mathrm{m}(\mathrm{P}-\mathrm{V})$ in the circumstance of constant tool offsets (conventional approach).

Although the case study presented here is for the grinding processes, the proposed controlling strategies based on the theoretical analysis of the contact deformation, could be applied for other manufacturing operations where both the tool and workpiece are compliant.

\section{Acknowledgements}

The authors acknowledge the supports from Natural Science Foundation of Zhejiang Province (LY20E050014, LQ20E050006), National Natural Science Foundation of China (51975302, 51805281), State Key Laboratory of Mechanical System and Vibration (No. MSV201908), Ningbo Science and Technology Bureau (2019A610154), and University of Nottingham Ningbo China (101180800099, 101190100001, 101180900005). Special thanks to Mr. W. Q. Zhu of the University of Nottingham Ningbo China for helpful discussions and technical assistance in performing the experiments, and Mr. Q. Liu of the University of Nottingham for helping scan the ground samples.

\section{Appendix A}

The grinding zone assumed as a perfect circular (with the radius of $a$ [37]) is discretized into $n$ annuluses in the radial direction and $m$ elements in the circumferential direction. The 
pressure is varied from different annuluses but same in one. Here, we neglect the pressure difference along the circumferential direction, and therefore, we focus on the element positions in the radial direction.

$$
\begin{gathered}
r_{c}^{i}=a / 2 n+a(i-1) / n, i=1,2,3, \ldots n \\
r_{n}^{i}=a(i-1) / n, i=1,2, \ldots n+1 \\
S_{i}=\pi a^{2}\left[i^{2}-(i-1)^{2}\right] / n^{2}, i=1,2, \ldots n \\
a_{c}=\sqrt{R_{2}^{2}-\left(R_{2}-e\right)^{2}} \\
a=\sqrt{\delta_{2} R_{2}}
\end{gathered}
$$

Please note that we establish the geometric equilibrium equation (Eq. (2)) concerning centroids and nodes of discretized elements, so the initial positions (Eq. (3)) are updated based on element positions $\left(r_{c}^{i}\right.$ and $\left.r_{n}^{i}\right)$. However, the resultant pressure is located at the element centroids.

\section{Appendix B}

Considering the auxiliary function $(\Phi)$ given by [34]

$$
\Phi=\sum_{i=1}^{n-1}\left(q_{i}-q_{i+1}\right)^{2}
$$

The mean square error is

$$
\psi\left(q_{i}\right)=\left(A_{j k} q_{k}-B_{j}\right)\left(A_{j i} q_{i}-B_{j}\right)+\lambda \Phi, k=1,2,3, \ldots n
$$

For a minimum of error, it is required that

$$
\partial \psi\left(q_{i}\right) / \partial q_{k}=2 A_{j i} A_{j k} q_{i}-2 A_{j k} B_{j}+\lambda \partial \Phi / \partial q_{k}=0, k=1,2,3, \ldots n
$$

where $\lambda$ is the FR control parameter for the pressure step of adjacent elements.

The matrix form of the Eq. (C.3) is given by

$$
[A]^{\top}[A]\{q\}+\lambda[\varphi]\{q\}=[A]^{\top}[B]
$$

where the diagonal matrix $[\varphi]$ is

$$
[\varphi]=\left[\begin{array}{rrrrrr}
1 & -1 & 0 & & & \\
-1 & 2 & -1 & & 0 & \\
0 & -1 & 2 & -1 & & \\
& & & \ddots & & \\
& 0 & & -1 & 2 & -1 \\
& & & & -1 & 1
\end{array}\right]
$$


Hence the contact pressure is

$$
\{q\}=\left([\mathrm{A}]^{\top}[\mathrm{A}]+\lambda[\varphi]\right)^{-1}[A]^{\top}[B]
$$

\section{Appendix C}

Partial derivatives of the thin wall deflection function, $\partial \delta_{1} / \partial r_{1}, \partial^{2} \delta_{1} / \partial r_{1}^{2}$, and $\partial^{2} \delta_{1} / \partial \theta^{2}$ in Eq. (26) and (27) are expressed as:

$$
\begin{gathered}
\partial \delta_{1} / \partial r_{1}=\left\{\begin{array}{l}
d \widehat{K}_{0} / d r_{1}+\sum_{m=1}^{\infty}\left(d \widehat{K}_{m} / d r_{1}\right) \cos m \theta, \text { for } 0 \leq r_{1} \leq b \\
d K_{0} / d r_{1}+\sum_{m=1}^{\infty}\left(d K_{m} / d r_{1}\right) \cos m \theta, \text { for } b \leq r_{1} \leq R_{1}
\end{array}\right. \\
\partial^{2} \delta_{1} / \partial r_{1}^{2}=\left\{\begin{array}{l}
d^{2} \widehat{K}_{0} / d r_{1}^{2}+\sum_{m=1}^{\infty}\left(d^{2} \widehat{K}_{m} / d r_{1}^{2}\right) \cos m \theta, \text { for } 0 \leq r_{1} \leq b \\
d^{2} K_{0} / d r_{1}^{2}+\sum_{m=1}^{\infty}\left(d^{2} K_{m} / d r_{1}^{2}\right) \cos m \theta, \text { for } b \leq r_{1} \leq R_{1}
\end{array}\right. \\
\partial^{2} \delta_{1} / \partial \theta^{2}=\left\{\begin{array}{l}
-\sum_{m=1}^{\infty} \widehat{K}_{m} m^{2} \cos m \theta, \text { for } 0 \leq r_{1} \leq b \\
-\sum_{m=1}^{\infty} K_{m} m^{2} \cos m \theta, \text { for } b \leq r_{1} \leq R_{1}
\end{array}\right.
\end{gathered}
$$

First-order derivative:

$$
\begin{gathered}
\left\{\begin{array}{c}
d \widehat{K}_{0} / d r_{1}=\operatorname{Pr}_{1}\left[2 \log \left(b / R_{1}\right)+\left(R_{1}^{2}-b^{2}\right) / R_{1}^{2}\right] / 8 \pi D_{1} \\
d \widehat{K}_{1} / d r_{1}=-P b^{3}\left[2\left(R_{1}^{2}-b^{2}\right) / R_{1}^{2} b^{2}+3\left(R_{1}^{2}-b^{2}\right)^{2} r_{1}^{2} / R_{1}^{4} b^{4}\right. \\
\left.-4 \log \left(R_{1} / b\right) / b^{2}\right] / 16 \pi D_{1} \\
d \widehat{K}_{m} / d r_{1}=P b^{m}\left\{m r_{1}^{m-1}\left[(m-1) b^{2}-m R_{1}^{2}+R_{1}^{2 m} / b^{2(m-1)}\right] / R_{1}^{2 m}\right. \\
+(m-1)(m+2) r_{1}^{m+1}\left[1-m b^{2} /(m+1) R_{1}^{2}\right. \\
\left.\left.-R_{1}^{2 m} /(m+1) b^{2 m}\right] / R_{1}^{2 m}\right\} / 8 m(m-1) \pi D_{1}
\end{array}\right. \\
\left\{\begin{array}{c}
d K_{0} / d r_{1}=P\left[2 r_{1} \log \left(r_{1} / R_{1}\right)+\left(r_{1}^{2}+b^{2}\right) / r_{1}-\left(R_{1}^{2}+b^{2}\right) r_{1} / R_{1}^{2}\right] / 8 \pi D_{1} \\
d K_{1} / d r_{1}=-P b^{3}\left[-1 / r_{1}^{2}+2\left(R_{1}^{2}-b^{2}\right) / R_{1}^{2} b^{2}-3\left(2 R_{1}^{2}-b^{2}\right) r_{1}^{2} / R_{1}^{4} b^{2}\right. \\
\left.-4 \log \left(R_{1} / r_{1}\right) / b^{2}+4 / b^{2}\right] / 16 \pi D_{1} \\
d K_{m} / d r_{1}=P b^{m}\left\{m r_{1}^{m-1}\left[(m-1) b^{2}-m R_{1}^{2}+(m-1) r_{1}^{2}\right] / R_{1}^{2 m}\right. \\
-m r_{1}^{m-1}\left[m(m-1) b^{2} r_{1}^{2} /(m+1) R_{1}^{2}\right] / R_{1}^{2 m} \\
+2(m-1) r_{1}^{m+1}\left(1-m b^{2} /(m+1) R_{1}^{2}\right) / R_{1}^{2 m} \\
-m\left[r_{1}^{2}-(m-1) b^{2} /(m+1)\right] / r_{1}^{m+1} \\
\left.+2 / r_{1}^{m-1}\right\} / 8 m(m-1) \pi D_{1}
\end{array}\right.
\end{gathered}
$$

Second-order derivative

$$
\left\{\begin{array}{c}
d^{2} \widehat{K}_{0} / d r_{1}^{2}=P\left[\log \left(b / R_{1}\right)+1-b^{2} / R_{1}^{2}\right] / 8 \pi D_{1} \\
d^{2} \widehat{K}_{1} / d r_{1}^{2}=-P b^{3}\left[6\left(R_{1}^{2}-b^{2}\right)^{2} r_{1} / R_{1}^{4} b^{4}\right] / 16 \pi D_{1} \\
d^{2} \widehat{K}_{m} / d r_{1}^{2}=P b^{m}\left\{m ( m - 1 ) r _ { 1 } ^ { m - 2 } \left[(m-1) b^{2}-m R_{1}^{2}\right.\right. \\
\left.+R_{1}^{2 m} / b^{2 m-2}\right] / R_{1}^{2 m}+(m-1)(m+2)(m+1) \\
r_{1}^{m} / R_{1}^{2 m}\left[1-m b^{2} /(m+1) R_{1}^{2}\right. \\
\left.\left.R_{1}^{2 m} /(m+1) b^{2 m}\right]\right\} / 8 m(m-1) \pi D_{1}
\end{array}\right.
$$




$$
\left\{\begin{array}{c}
d^{2} K_{0} / d r_{1}^{2}=P\left[2 \log \left(r_{1} / R_{1}\right)+2-b^{2} / r_{1}^{2}-b^{2} / R_{1}^{2}\right] / 8 \pi D_{1} \\
d^{2} K_{1} / d r_{1}^{2}=-P b^{3}\left[2 / r_{1}^{3}-6\left(2 R_{1}^{2}-b^{2}\right) r_{1} / R_{1}^{4} b^{2}+4 r_{1} / b^{2}\right] / 16 \pi D_{1} \\
d^{2} K_{m} / d r_{1}^{2}=P b^{m}\left\{m ( m - 1 ) r _ { 1 } ^ { m - 2 } \left[(m-1) b^{2}-m R_{1}^{2}+(m-1) r_{1}^{2}\right.\right. \\
\left.-m(m-1) b^{2} r_{1}^{2} /(m+1) R_{1}^{2}\right] / R_{1}^{2 m} \\
+2(m-1)(2 m+1) r_{1}^{m}\left[1-m b^{2} /(m+1) R_{1}^{2}\right] / R_{1}^{2 m} \\
+(2-m)(1-m) / r_{1}^{m} \\
\left.-m(m-1) b^{2} / r_{1}^{m+2}\right\} / 8 m(m-1) \pi D_{1}
\end{array}\right.
$$

\section{References}

[1] A. Spampinato, D.A. Axinte, On modelling the interaction between two rotating bodies with statistically distributed features: an application to dressing of grinding wheels, Proc. R. Soc. A Math. Phys. Eng. Sci. 473 (2017) 20170466. doi:10.1098/rspa.2017.0466.

[2] A. Lu, T. Jin, Q. Liu, Z. Guo, M. Qu, H. Luo, M. Han, Modeling and prediction of surface topography and surface roughness in dual-axis wheel polishing of optical glass, Int. J. Mach. Tools Manuf. 137 (2019) 13-29. doi:10.1016/j.ijmachtools.2018.10.001.

[3] C. Li, X. Li, Y. Wu, F. Zhang, H. Huang, Deformation mechanism and force modelling of the grinding of YAG single crystals, Int. J. Mach. Tools Manuf. 143 (2019) 23-37. doi:10.1016/j.ijmachtools.2019.05.003.

[4] Z. Liao, A. Abdelhafeez, H. Li, Y. Yang, O.G. Diaz, D. Axinte, State-of-the-art of surface integrity in machining of metal matrix composites, Int. J. Mach. Tools Manuf. 143 (2019) 63-91. doi:10.1016/j.ijmachtools.2019.05.006.

[5] H.N. Li, Y. Yang, Y.J. Zhao, Z. Zhang, W. Zhu, W. Wang, H. Qi, On the periodicity of fixed-abrasive planetary lapping based on a generic model, J. Manuf. Process. 44 (2019) 271-287. doi:10.1016/j.jmapro.2019.05.036.

[6] J.N. Reddy, Theory and analysis of elastic plates and shells, CRC press, 2006.

[7] T. Wang, C. Wu, H. Liu, M. Chen, J. Cheng, Z. Fang, B. Yu, Configuration design and accuracy analysis of special grinding machine for thin-walled small concave surfaces, Precis. Eng. 56 (2019) 293-302. doi:10.1016/j.precisioneng.2018.12.009.

[8] X. Wang, Z. Li, Q. Bi, L. Zhu, H. Ding, An accelerated convergence approach for realtime deformation compensation in large thin-walled parts machining, Int. J. Mach. Tools Manuf. 142 (2019) 98-106. doi:10.1016/j.ijmachtools.2018.12.004.

[9] S. Ratchev, S. Liu, W. Huang, A.A. Becker, Milling error prediction and compensation 
in machining of low-rigidity parts, Int. J. Mach. Tools Manuf. 44 (2004) 1629-1641. doi:10.1016/j.ijmachtools.2004.06.001.

[10] I. Lazoglu, A. Mamedov, Deformation of thin parts in micromilling, CIRP Ann. 65 (2016) 117-120. doi:10.1016/j.cirp.2016.04.077.

[11] S. Zhang, Q. Bi, Y. Ji, Y. Wang, Real-time thickness compensation in mirror milling based on modified Smith predictor and disturbance observer, Int. J. Mach. Tools Manuf. 144 (2019) 103427. doi:10.1016/j.jimachtools.2019.103427.

[12] Q. Bi, N. Huang, S. Zhang, C. Shuai, Y. Wang, Adaptive machining for curved contour on deformed large skin based on on-machine measurement and isometric mapping, Int. J. Mach. Tools Manuf. 136 (2019) 34-44. doi:10.1016/j.jimachtools.2018.09.001.

[13] S. Ratchev, S. Liu, A.A. Becker, Error compensation strategy in milling flexible thinwall parts, J. Mater. Process. Technol. 162-163 (2005) 673-681. doi:10.1016/j.jmatprotec.2005.02.192.

[14] J. Ma, G. He, Z. Liu, F. Qin, S. Chen, X. Zhao, Instantaneous cutting-amount planning for machining deformation homogenization based on position-dependent rigidity of thin-walled surface parts, J. Manuf. Process. 34 (2018) 401-411. doi:10.1016/j.jmapro.2018.05.027.

[15] S. Wimmer, M.F. Zaeh, The Prediction of Surface Error Characteristics in the Peripheral Milling of Thin-Walled Structures, J. Manuf. Mater. Process. 2 (2018) 13. doi:10.3390/jmmp2010013.

[16] F. Chen, H. Zhao, D. Li, L. Chen, C. Tan, H. Ding, Robotic grinding of a blisk with two degrees of freedom contact force control, Int. J. Adv. Manuf. Technol. 101 (2018) 461-474. doi:10.1007/s00170-018-2925-6.

[17] Z.-C. Cao, C.F. Cheung, Multi-scale modeling and simulation of material removal characteristics in computer-controlled bonnet polishing, Int. J. Mech. Sci. 106 (2016) 147-156. doi:10.1016/j.jjmecsci.2015.12.011.

[18] A. Beaucamp, Y. Namba, H. Combrinck, P. Charlton, R. Freeman, Shape adaptive grinding of CVD silicon carbide, CIRP Ann. 63 (2014) 317-320. doi:10.1016/j.cirp.2014.03.019.

[19] A. Beaucamp, Y. Namba, P. Charlton, Process mechanism in shape adaptive 
grinding (SAG), CIRP Ann. 64 (2015) 305-308. doi:10.1016/j.cirp.2015.04.096.

[20] A. Beaucamp, P. Simon, P. Charlton, C. King, A. Matsubara, K. Wegener, Brittleductile transition in shape adaptive grinding (SAG) of SiC aspheric optics, Int. J. Mach. Tools Manuf. 115 (2017) 29-37. doi:10.1016/j.jjmachtools.2016.11.006.

[21] C.F. Cheung, L.B. Kong, L.T. Ho, S. To, Modelling and simulation of structure surface generation using computer controlled ultra-precision polishing, Precis. Eng. 35 (2011) 574-590. doi:10.1016/j.precisioneng.2011.04.001.

[22] W.-L. Zhu, A. Beaucamp, Ultra-precision finishing of low expansion ceramics by compliant abrasive technologies: A comparative study, Ceram. Int. 45 (2019) 1152711538. doi:10.1016/j.ceramint.2019.03.022.

[23] A. Beaucamp, Y. Namba, I. Inasaki, H. Combrinck, R. Freeman, Finishing of optical moulds to $N / 20$ by automated corrective polishing, CIRP Ann. 60 (2011) 375-378. doi:10.1016/j.cirp.2011.03.110.

[24] A. Beaucamp, Y. Namba, Super-smooth finishing of diamond turned hard X-ray molding dies by combined fluid jet and bonnet polishing, CIRP Ann. 62 (2013) 315318. doi:10.1016/j.cirp.2013.03.010.

[25] W.-L. Zhu, Y. Yang, H.N. Li, D. Axinte, A. Beaucamp, Theoretical and experimental investigation of material removal mechanism in compliant shape adaptive grinding process, Int. J. Mach. Tools Manuf. 142 (2019) 76-97. doi:10.1016/j.ijmachtools.2019.04.011.

[26] W.-L. Zhu, B. Anthony, Investigation of critical material removal transitions in compliant machining of brittle ceramics, Mater. Des. (2019) 108258. doi:10.1016/j.matdes.2019.108258.

[27] R. Pan, B. Zhong, D. Chen, Z. Wang, J. Fan, C. Zhang, S. Wei, Modification of tool influence function of bonnet polishing based on interfacial friction coefficient, Int. J. Mach. Tools Manuf. 124 (2018) 43-52. doi:10.1016/j.ijmachtools.2017.09.003.

[28] B. Zhong, H. Huang, X. Chen, J. Wang, R. Pan, Z. Wen, Impact of pad conditioning on the bonnet polishing process, Int. J. Adv. Manuf. Technol. 98 (2018) 539-549. doi:10.1007/s00170-018-2250-0.

[29] X. Xu, D. Zhu, H. Zhang, S. Yan, H. Ding, TCP-based calibration in robot-assisted belt grinding of aero-engine blades using scanner measurements, Int. J. Adv. Manuf. 
Technol. 90 (2017) 635-647. doi:10.1007/s00170-016-9331-8.

[30] G. Xiao, Y. Huang, Equivalent self-adaptive belt grinding for the real- $R$ edge of an aero-engine precision-forged blade, Int. J. Adv. Manuf. Technol. 83 (2016) 16971706. doi:10.1007/s00170-015-7680-3.

[31] B.A. Galanov, I.K. Valeeva, Sliding adhesive contact of elastic solids with stochastic roughness, Int. J. Eng. Sci. 101 (2016) 64-80. doi:10.1016/j.jjengsci.2015.12.007.

[32] S.P. Timoshenko, S. Woinowsky-Krieger, Theory of plates and shells, McGraw-hill, 1959.

[33] V.L. Popov, Contact mechanics and friction, Berlin: Springer Berlin Heidelberg, 2010.

[34] K.P. Singh, Contact Stresses in Elastic Bodies With Arbitrary Profiles, 1972.

[35] K.P. Singh, B. Paul, Numerical Solution of Non-Hertzian Elastic Contact Problems, J. Appl. Mech. 41 (1974) 484-490. doi:10.1115/1.3423314.

[36] K.P. SINGH, B. PAUL, A mehtod for solving ill posed integral equations of the first kind, Comput. Methods Appl. Mech. Eng. 2 (1973) 339-348.

[37] K.L. Johnson, Contact mechanics, Cambridge university press, 1987.

[38] A.N. Gent, On the relation between indentation hardness and Young's modulus, Rubber Chem. Technol. 31 (1958) 896-906. 